\title{
Spectra and Line Profiles of FU Orionis Objects: Comparisons between Boundary Layer Models and Observations
}

\section{Citation}

Popham, Robert, Scott Kenyon, Lee Hartmann, and Ramesh Narayan. 1996. "Spectra and Line Profiles of FU Orionis Objects: Comparisons between Boundary Layer Models and Observations." The Astrophysical Journal 473 (1): 422-36. https://doi.org/10.1086/178155.

\section{Permanent link}

http://nrs.harvard.edu/urn-3:HUL.InstRepos:41384906

\section{Terms of Use}

This article was downloaded from Harvard University's DASH repository, and is made available under the terms and conditions applicable to Other Posted Material, as set forth at http:// nrs.harvard.edu/urn-3:HUL.InstRepos:dash.current.terms-of-use\#LAA

\section{Share Your Story}

The Harvard community has made this article openly available. Please share how this access benefits you. Submit a story.

Accessibility 


\title{
SPECTRA AND LINE PROFILES OF FU ORIONIS OBJECTS: COMPARISONS BETWEEN BOUNDARY LAYER MODELS AND OBSERVATIONS
}

\author{
Robert Popham, Scott Kenyon, Lee Hartmann, and Ramesh Narayan \\ Harvard-Smithsonian Center for Astrophysics
}

\begin{abstract}
We present solutions for the accretion disks and boundary layers in pre-main-sequence stars undergoing FU Orionis outbursts. These solutions differ from earlier disk solutions in that they include a self-consistent treatment of the boundary layer region. In a previous paper (Popham 1996), we showed that these stars should stop accreting angular momentum once they spin up to modest rotation rates. Here we show that for reasonable values of $\alpha$, these low angular momentum accretion rate solutions fit the spectra and line profiles observed in FU Orionis objects better than solutions with high rates of angular momentum accretion. We find solutions which fit the observations of FU Orionis and V1057 Cygni. These solutions have mass accretion rates of 2 and $1 \times 10^{-4} \mathrm{M}_{\odot} \mathrm{yr}^{-1}$, stellar masses of 0.7 and $0.5 \mathrm{M}_{\odot}$, and stellar radii of 5.75 and $5.03 \mathrm{R}_{\odot}$, respectively. They also have modest stellar rotation rates $8-9 \times 10^{-6} \mathrm{~s}^{-1}$, comparable to the observed rotation rates of $\mathrm{T}$ Tauri stars, and angular momentum accretion rates of zero. This supports our earlier suggestion that FU Orionis outbursts may regulate the rotation rates of $\mathrm{T}$ Tauri stars.
\end{abstract}

Subject headings: accretion, accretion disks - stars: formation-stars: pre-main-sequence- stars: rotation

\section{Introduction}

The FU Orionis objects are a class of accreting pre-main-sequence stars which are undergoing dramatic outbursts in their brightness (see Hartmann, Kenyon, \& Hartigan 1993; Kenyon 1995; Hartmann \& Kenyon 1996 for reviews). These outbursts are believed to result from a sudden, large increase in the mass accretion rate in an accretion disk around a T Tauri star. Line profiles in FU Orionis systems are generally double-peaked, as expected from a rotating disk (Hartmann \& Kenyon 1985, 1987). The observed variation 
in linewidths as a function of temperature also suggests a disk origin; line profiles in the optical are broader than those in the infrared. This is the trend expected for lines produced in a Keplerian disk, where the rotational velocity and temperature both decrease with radius, so that smaller rotational linewidths are produced in cooler regions (Hartmann \& Kenyon 1987). The spectral energy distributions of these systems are also quite broad, which is consistent with a disk with a range of effective temperatures at different radii.

Accretion disk models have been successful in reproducing observations of individual systems. Kenyon, Hartmann, \& Hewett (1988, hereafter KHH) constructed steady thin disk models for FU Orionis and V1057 Cygni. They found that simple thin disk models were able to reproduce both line profiles and broad-band spectra of these systems. These models had mass accretion rates of about $10^{-4} \mathrm{M}_{\odot} \mathrm{yr}^{-1}$, stellar masses of $0.3-1.0 \mathrm{M}_{\odot}$, and stellar radii of $4-6 \mathrm{R}_{\odot}$. They had Keplerian rotational velocities, and maximum disk temperatures of $7200 \mathrm{~K}$ for FU Orionis and $6590 \mathrm{~K}$ for V1057 Cygni.

One major simplifying assumption made by the KHH models is that the accretion disks in FU Orionis systems can be approximated by a standard Keplerian thin disk model. This assumption breaks down in the inner disk, where the transition from the disk to the accreting star occurs in the boundary layer region. Early estimates for the temperature of the boundary layer region suggested that it would be much hotter than permitted by observations (Hartmann \& Kenyon 1985; Kenyon et al. 1989). These estimates were made by assuming that the boundary layer luminosity is half of the total accretion luminosity, and that it is radiated from a small region of the inner disk, with a radial extent of a few percent of the stellar radius. These large luminosities radiated from such a small area would produce boundary layer temperatures approaching 30,000 K, which would mean that the boundary layer would emit large ultraviolet fluxes. IUE observations of FU Orionis objects by Kenyon et al. (1989) demonstrated that these large fluxes were not present. Kenyon et al. (1989) suggested three possible reasons why the boundary layer might not be as hot as predicted by these simple estimates. First, some of the boundary layer energy might be going into expanding the central star. Second, the central star might be rotating rapidly due to accretion spinup. Third, the disk might not be thin, and so the radial extent of the boundary layer might be much larger than a few percent of the stellar radius.

Recently, we have begun to calculate self-consistent solutions for the structure of boundary layers in FU Orionis systems. These solutions suggest that all of the mechanisms mentioned by Kenyon et al. (1989) act to decrease the effective temperature of the boundary layer below the temperatures suggested by simple estimates. Popham et al. (1993, hereafter PNHK) obtained boundary layer solutions for pre-main-sequence stellar parameters and mass accretion rates $\dot{M}$ ranging from $10^{-7}-10^{-4} \mathrm{M}_{\odot} \mathrm{yr}^{-1}$. They showed that at the higher 
accretion rates, which correspond to FU Orionis systems, the radial extent of the boundary layer region becomes quite large, and the boundary layer temperature drops much closer to that of the inner disk. Thus as $\dot{M}$ increases it becomes progressively more difficult to distinguish the contribution of the boundary layer region to the overall spectrum. PNHK also showed that in high- $\dot{M}$ systems, the accreting material is quite hot when it reaches the stellar radius, with a temperature which is a substantial fraction of the virial temperature. This means that a substantial fraction of the accretion luminosity is advected into the star, decreasing the luminosity radiated by the boundary layer. PNHK calculated two models with $\dot{M}=10^{-4} \mathrm{M}_{\odot} \mathrm{yr}^{-1}$, one with $\alpha=10^{-1}$ and the other with $\alpha=10^{-3}$. The $\alpha=10^{-1}$ solution reached a peak $T_{\text {eff }} \simeq 17,000 \mathrm{~K}$, with $H / R \sim 0.2$, while the $\alpha=10^{-3}$ solution had a lower peak $T_{\text {eff }} \simeq 9500 \mathrm{~K}$ and $H / R \sim 0.4$. Godon (1996) confirmed these results with a time-dependent model, finding a peak $T_{\text {eff }}$ of $14,000 \mathrm{~K}$ and $H / R \simeq 0.40$ for $\dot{M}=10^{-4} \mathrm{M}_{\odot} \mathrm{yr}^{-1}$ and $\alpha=0.3$.

Recently, Popham (1996, hereafter Paper I) presented boundary layer solutions for FU Orionis parameters, and examined the effect of FU Orionis outbursts on the spin evolution of the underlying T Tauri stars. Event statistics suggest that the outbursts may dominate the mass accretion onto T Tauri stars (Hartmann \& Kenyon 1996); if so, they should also dominate the angular momentum accretion. Disk accretion generally adds angular momentum to the accreting star and spins it up; however, when the accreting star nears breakup, the angular momentum accretion rate drops rapidly and becomes negative, so that the accreting star stops spinning up (Popham \& Narayan 1991; Paczyński 1991). Paper I showed that for the disk parameters which characterize FU Orionis systems, the accreting star stops spinning up once it reaches $20-40 \%$ of breakup speed (i.e., the Keplerian rotation rate at the stellar surface). The star can then continue to accrete mass while maintaining an equilibrium rotation rate. Paper I suggested that these low angular momentum accretion rate solutions, obtained during outbursts, play an important role in maintaining the observed slow rotation rates of $\mathrm{T}$ Tauri stars.

The boundary layer region should make an important contribution to the observed spectral energy distribution and spectral line profiles of FU Orionis systems. In the boundary layer, the rotational velocity of the accreting material, which determines the linewidths, varies rapidly with radius. It drops from its disk value, generally close to Keplerian, to the surface rotational velocity of the star, which is usually much slower. As the material loses its rotational kinetic energy, up to half of the accretion luminosity is released in the boundary layer region. Since disk models which include the boundary layer will have quite different temperature and rotational velocity profiles from Keplerian thin disk models, they will produce somewhat different spectra and line profiles. Also, as shown in Paper I, low angular momentum accretion rate solutions have different temperature and 
rotational velocity profiles from those with high angular momentum accretion rates. This raises several questions: 1) whether solutions which include the boundary layer region will be able to match the observations of FU Orionis systems; 2) which types of boundary layer solutions will match the observations, and 3) how the inclusion of the boundary layer will change the model parameters inferred from Keplerian disk solutions.

In this paper, we attempt to answer these questions by calculating boundary layer solutions for FU Orionis systems, and comparing the spectra and line profiles produced by these solutions to the observations. In $\S 2$, we briefly describe our boundary layer model and the procedures we use for calculating spectra and line profiles from our solutions. In $\S 3$, we discuss the general characteristics of the spectra and profiles produced by our boundary layer solutions. We show how these characteristics depend on solution parameters such as the mass accretion rate, the stellar mass, radius, and rotation rate, the angular momentum accretion rate, and the viscosity parameter $\alpha$. We compare our solutions to observations of the spectra and line profiles of individual FU Orionis systems in $\S 4$, and discuss our results and their implications in $\S 5$. $\S 6$ gives a summary.

\section{Boundary Layer and Disk Model}

The model we use to calculate the structure of the boundary layer and accretion disk is identical to the one used in Paper I and very similar to the model used in several earlier papers (Narayan \& Popham 1993; PNHK; Popham \& Narayan 1995), where it is described in detail. The model uses the steady-state, axisymmetric slim disk equations originally

developed by Paczyǹski and collaborators for modeling disks around black holes (Paczyǹski \& Bisnovatyi-Kogan 1981; Muchotrzeb \& Paczyǹski 1982; Abramowicz et al. 1988) to describe the radial structure of the disk. The slim disk equations dispense with some of the assumptions of the standard thin disk model (Shakura \& Sunyaev 1973), and include terms which are assumed to be small in the thin disk formulation. These include the radial pressure gradient and acceleration in the radial momentum equation, and radial energy transport by radiation and advection. Also, while the thin disk equations assume that the angular momentum accretion rate takes on a standard value, the slim disk equations allow for different angular momentum accretion rates. The additional terms in the slim disk equations involve radial derivatives which make them differential equations requiring a numerical solution. We use approximate relations to describe the vertical structure of the disk. We solve the equations using a relaxation method, on a 1001-point radial grid which extends from $R_{*}$ to $100 R_{*}$, with grid points concentrated in the inner boundary layer region. 


\subsection{Spectra}

Our solutions yield the effective temperature of the disk surface as a function of radius. From this, we construct spectra by simply adding up the contributions from each disk annulus. We use two different methods for calculating the spectrum of each annulus. The first is simply to assume that the disk surface emits as a blackbody at the local effective temperature. This has the advantages of being simple and independent of any assumptions about the vertical structure of the disk. The second method is to use a grid of stellar atmospheres, using the methods described by Hartmann \& Kenyon (1985). This should do a better job of reproducing features such as the Balmer jump; however, it is still only approximate, since the disk atmosphere probably differs somewhat from a stellar atmosphere.

We assume that the only effect of inclination is to change the projected area of the disk as seen by an observer. Thus, the observed flux from the disk is $F_{\lambda}=L_{\lambda} \cos i / 2 \pi d^{2}$, where $L_{\lambda}$ is the total disk luminosity, $i$ is the inclination angle, and $d$ is the distance. At the average value of $\langle\cos i\rangle=1 / 2$, we have the standard expression $F_{\lambda}=L_{\lambda} / 4 \pi d^{2}$.

\subsection{Line Profiles}

In order to calculate spectral line profiles from our models, we begin by calculating the line profile produced by a single annulus of the disk. We take the profile function for a rotating disk, $\Phi(\Delta v)=\left[1-\left(\Delta v / v_{\text {los }}\right)^{2}\right]^{-1 / 2}$, where $\Delta v$ is the deviation in velocity from line center, and $v_{l o s}=v_{\text {rot }} \sin i$ is the projected rotational velocity at inclination angle $i$. We convolve this profile function with a Gaussian broadening function $\exp \left[-\left(\Delta v / v_{b}\right)^{2}\right]$, where $v_{b}$ is the instrumental broadening due to the resolution of the spectrograph.

Next, we sum up the line profile contributions from all disk annuli. We weight the annuli according to their contribution to the blackbody flux at the wavelength of the spectral line. This is equivalent to assuming that the equivalent width of the line stays constant as the effective temperature varies. For the lines we consider, metal lines at 6170 $\AA$ and CO lines at $2.2 \mu \mathrm{m}$, this is a reasonable assumption, except at high temperatures, where the species in question disappear. For this reason, we set the weight to zero when the effective temperature exceeds a cutoff value, which we take to be $8000 \mathrm{~K}$ for the $6170 \AA$ lines and $5000 \mathrm{~K}$ for the $2.2 \mu \mathrm{m}$ lines. 


\section{Spectra and Line Profiles from Boundary Layer Solutions and Their Dependence on Solution Parameters}

Our disk and boundary layer solutions have a number of free parameters which can affect their spectra and line profiles. These include the usual parameters used to characterize accreting systems: the mass accretion rate $\dot{M}$, and the stellar mass and radius $M_{*}$ and $R_{*}$. As discussed in Paper I, even when these parameters are specified, there are still a range of solutions available, which are characterized by different values of the stellar rotation rate $\Omega_{*}$ and the disk height at the stellar radius $H_{*}$, which then specify the angular momentum accretion rate $\dot{J}$. Finally, one can specify the parameter $\alpha$, which characterizes the magnitude of the viscosity.

With such a wide range of solution parameters available, we begin by examining the effects of each parameter on our model spectra and line profiles. We then compare these to the general observed characteristics of FU Orionis systems in order to narrow down the parameter space in which solutions in reasonable agreement with the data will be found. Later in $\S 4$, we will attempt to find solutions which match the observations of individual systems (FU Orionis and V1057 Cygni).

The general observed characteristics of FU Orionis systems, which we will use to try to limit the parameter space for our solutions, are as follows: the line profiles are generally double-peaked, both in the optical and the infrared (Hartmann \& Kenyon 1985, 1987). The line widths vary widely from system to system; some are below the instrumental resolution of $10-15 \mathrm{~km} \mathrm{~s}^{-1}$, while others have FWHM values in excess of $100 \mathrm{~km} \mathrm{~s}^{-1}$. Such a range is not surprising, since the linewidth depends on the projected rotational velocity $v_{\text {rot }} \sin i$. The linewidths in the optical are broader than those in the infrared; linewidths measured at $6170 \AA$ are $\sim 40 \%$ larger than those measured at $2.2 \mu \mathrm{m}$.

The dereddened broad-band spectra of FU Orionis systems generally have their peak $\lambda F_{\lambda}$ values in the red part of the spectrum (KHH). The spectra fall off quite rapidly at blue and ultraviolet wavelengths, with $\lambda F_{\lambda}$ in the $\mathrm{U}$ band a factor of three or more smaller than the peak value. The best estimates for the distances of these systems give total luminosities of a few hundred $\mathrm{L}_{\odot}$ (Bell \& Lin 1994; Hartmann \& Kenyon 1996).

\subsection{Standard Accretion Parameters: $\dot{M}, M_{*}$, and $R_{*}$}

The three most fundamental parameters describing an accretion disk are the mass accretion rate $\dot{M}$ and the mass $M_{*}$ and radius $R_{*}$ of the accreting star. In the thin disk formulation, these parameters can be combined to derive three standard quantities which 
characterize the spectra and linewidths produced by the disk: the accretion luminosity $L_{a c c}=G \dot{M} M_{*} / R_{*}$, the maximum disk temperature $T_{\max }=0.287\left(G \dot{M} M_{*} / \sigma R_{*}^{3}\right)^{1 / 4}$, and the Keplerian rotational velocity at $R_{*}, v_{K}\left(R_{*}\right)=\left(G M_{*} / R_{*}\right)^{1 / 2}$. These quantities provide a good general guide to the variations in the spectra and line profiles that result from variations in $\dot{M}, M_{*}$, and $R_{*}$. The accretion luminosity characterizes the brightness (the vertical position) of the spectrum, the maximum temperature controls the peak wavelength (the horizontal position) of the spectrum, and the Keplerian rotational velocity at $R_{*}$ characterizes the width of a line profile.

For a standard thin accretion disk, the quantities $L_{a c c}, T_{m a x}$, and $v_{K}\left(R_{*}\right)$ represent the exact values of the luminosity and the maximum effective temperature and rotational velocity of the disk. In our models, the situation is more complex, for two reasons. First, we include the boundary layer region in our solutions. The effects of variations in $\dot{M}, M_{*}$ and $R_{*}$ upon the boundary layer are more complex than their effects upon the disk, and the boundary layer is also affected by other parameters such as the rotation rate of the accreting star $\Omega_{*}$ and the angular momentum accretion rate. Second, our slim disk equations include important terms, described in $\S 2$, which are not included in the standard thin disk model. Thus, for our solutions, $L_{a c c}, T_{m a x}$, and $v_{K}\left(R_{*}\right)$ are only characteristic values which provide a good approximate description of the outer parts of the disk, but not of the inner portions close to the star. The radiated luminosity of our solutions can differ substantially from $L_{a c c}$, since energy can be advected into the star, and variations in $\Omega_{*}$ change the boundary layer luminosity. The boundary layer region is usually hotter than $T_{\max }$, and the rotational velocity of the disk is often well below Keplerian there.

As an example of the changes in the boundary layer structure produced by variations in $\dot{M}$ and $R_{*}$, we show in Figure 1 three solutions with the parameters used in Paper I. The first solution has $\dot{M}=10^{-4.3} \mathrm{M}_{\odot} \mathrm{yr}^{-1}, R_{*}=2.25 \times 10^{11} \mathrm{~cm}$, the second has $\dot{M}=10^{-4.15} \mathrm{M}_{\odot} \mathrm{yr}^{-1}$, $R_{*}=3 \times 10^{11} \mathrm{~cm}$, and the third $\dot{M}=10^{-4.0} \mathrm{M}_{\odot} \mathrm{yr}^{-1}, R_{*}=4 \times 10^{11} \mathrm{~cm}$. Note that these choices of parameters keep the accretion luminosity $L_{a c c}$ approximately constant at $\simeq 10^{36} \mathrm{ergs} \mathrm{s}^{-1} \simeq 250 \mathrm{~L}_{\odot}$, since the ratio $\dot{M} / R_{*}$ stays nearly constant. The maximum disk temperature $T_{\max }$ and the Keplerian rotational velocity at the stellar radius $v_{K}\left(R_{*}\right)$ both decrease as $\dot{M}$ and $R_{*}$ increase. All three solutions have $M_{*}=0.5 \mathrm{M}_{\odot}$, and $\alpha=10^{-2}$, and all three use the boundary condition $v_{R, *}=-1000 \mathrm{~cm} \mathrm{~s}^{-1}$, which will be discussed below. These are high angular momentum accretion rate solutions. Throughout this work, we express the angular momentum accretion rate $\dot{J}$ as $j \equiv \dot{J} / \dot{M} \Omega_{K}\left(R_{*}\right) R_{*}^{2}$, so that $j=1$ for a standard thin disk solution. The solutions in Fig. 1 have $j=1.0,0.9$, and 0.8 , respectively.

Figure 1a shows the angular velocity $\Omega$ for these solutions. All three have a peak in $\Omega$; inside the peak, $\Omega$ drops to $\Omega_{*} \ll \Omega_{K}\left(R_{*}\right)$, while outside the peak, $\Omega$ decreases at a roughly 
Keplerian rate. The main feature we wish to emphasize is that the location of the peak changes from about $1.8 R_{*}$ to $2.6 R_{*}$ as $\dot{M}$ increases; thus the dynamical boundary layer width doubles from $0.8 R_{*}$ to $1.6 R_{*}$ as $\dot{M}$ doubles. Another important feature is that as $\dot{M}$ increases, $\Omega$ drops farther below $\Omega_{K}$, indicated by a dashed line. At $\dot{M}=10^{-4.0} \mathrm{M}_{\odot} \mathrm{yr}^{-1}$, the peak value of $\Omega$ is only half of $\Omega_{K}$ at that radius. This is caused by the increase in pressure support at large values of $\dot{M}$. Figure 1 b shows the effective temperatures of these solutions. All three reach peak values just outside of $R_{*}$, but the peak is much more pronounced at lower $\dot{M}$. This is not surprising, since the dissipation of energy is much more concentrated in the smaller boundary layer. As $\dot{M}$ increases, the peak in $T_{e f f}$ due to the boundary layer dissipation becomes broader until it becomes difficult to distinguish it from the $T_{\text {eff }}$ profile of the disk. This broadening and cooling of the boundary layer with increasing $\dot{M}$ was pointed out in our earlier paper (PNHK) for $\dot{M}$ ranging from $10^{-7}$ to $10^{-4} \mathrm{M}_{\odot} \mathrm{yr}^{-1}$. Fig. 1 shows that at the upper end of this range, a factor of two increase in $\dot{M}$ can make a qualitative difference in the appearance of the boundary layer region.

The $6170 \AA$ line profiles for these solutions are shown in Figure 1c. These profiles reflect the variations in the rotational velocities in the inner portion of the disk. All three profiles are centrally peaked, due to the presence of the hot, slowly rotating material in the boundary layer region. At the lowest $\dot{M}$, the rapidly rotating inner disk produces a double-peaked component, which is filled in by the contribution from the more slowly rotating boundary layer, leaving broad shoulders on the combined line profile. As the boundary layer extends to larger radii at higher values of $\dot{M}$, the double-peaked component becomes even more difficult to distinguish; it only appears as wings on the centrally-peaked boundary layer profile. The width of the profiles decreases dramatically as $\dot{M}$ and $R_{*}$ increase, since the peak rotational velocity is much smaller. None of the profiles resemble the double-peaked profiles observed in FU Orionis systems; clearly the inclusion of the boundary layer makes a crucial difference in the overall line profile by reducing the rotational velocities over a wide region of the inner disk, where the disk is brightest.

Figure 1d shows blackbody spectra of these solutions. They have nearly the same luminosity, but the spectrum moves to longer wavelengths as $\dot{M}$ increases: the peak $\lambda F_{\lambda}$ moves from $\sim 0.5 \mu \mathrm{m}$ to $\sim 0.75 \mu \mathrm{m}$. This change in peak wavelength is larger than would be expected from the change in the $T_{\max }$ of the disk, due to the decrease in the boundary layer effective temperature. Separate boundary layer and disk components are not distinguishable in the spectrum, as they are at lower values of $\dot{M}$ (PNHK). 


\subsection{Stellar Rotation Rate, Disk Height, and Angular Momentum Accretion Rate: $\Omega_{*}, H_{*}$, and $j$}

In Paper I, we showed that a wide range of boundary layer solutions can be found for a single set of values of $\dot{M}, M_{*}$, and $R_{*}$, These solutions have different values of the stellar rotation rate $\Omega_{*}$ and the disk height at the stellar radius $H_{*}$, and they also have different values of the angular momentum accretion rate $j$. Note that only two of these three variables are independent, so we can specify a unique solution by choosing the values of two of them. We plotted the locations of these solutions in the $\Omega_{*}-H_{*}$ plane as lines of constant $j$. We found that solutions with high angular momentum accretion rates $j \sim 1$ formed Z-shaped tracks which fell in the low- $\Omega_{*}$ or the low- $H_{*}$ regions of the plane, as illustrated in Figure 2 for solutions with $j=0.9, \dot{M}=10^{-4.15} \mathrm{M}_{\odot} \mathrm{yr}^{-1}, M_{*}=0.5 \mathrm{M}_{\odot}$, $R_{*}=3 \times 10^{11} \mathrm{~cm}$, and $\alpha=0.01$. We were also able to find solutions with small or negative angular momentum accretion rates, $j \lesssim 0$. These solutions have moderate values of both $\Omega_{*}$ and $H_{*}$, as shown by the $j=0$ and $j=-1$ tracks in Figure 2. How do the spectra and line profiles produced by these two types of solutions compare to the data?

We begin by considering high- $j$ solutions. Figure 3 shows a set of solutions with $j=0.9$ which sit on the Z-shaped track in Figure 2. They have different values of $\Omega_{*}$ and $H_{*}$. The low $-H_{*}$ solutions have a narrow boundary layer, as evidenced by the pronounced peaks in both $\Omega$ and $T_{\text {eff }}$ close to $R_{*}$. The high- $H_{*}$ solutions have much broader boundary layers, and much flatter $\Omega$ and $T_{\text {eff }}$ profiles. These are reflected in very different line profiles and spectra; the high- $H_{*}$ solutions have strongly centrally peaked narrow profiles as a result of their small rotational velocities throughout the wide boundary layer. As $H_{*}$ decreases, the rotational velocity in the inner disk increases, and the boundary layer gets smaller; thus the profiles get wider, and the double-peaked disk component becomes more prominent, forming a triple-peaked profile at $H_{*}=0.9 \times 10^{11} \mathrm{~cm}$ and finally a double-peaked profile at $H_{*}=0.8 \times 10^{11} \mathrm{~cm}$. The spectra produced by these solutions also vary substantially with $H_{*}$; as $H_{*}$ decreases, the smaller boundary layer reaches higher peak effective temperatures and produces bluer spectra. None of the high- $j$ solutions agrees with the observed spectra and line profiles of FU Orionis systems. Only the low- $H_{*}$ solutions produce reasonably double-peaked profiles, but their spectra are too blue. The $H_{*}=0.9 \times 10^{11} \mathrm{~cm}$ solution peaks around $0.5 \mu \mathrm{m}$; the $H_{*}=0.8 \times 10^{11} \mathrm{~cm}$ solution actually peaks in the red, but is still fairly bright in the blue and ultraviolet, unlike the observed systems.

Note that the $H_{*}=0.8 \times 10^{11} \mathrm{~cm}$ solution has both a lower luminosity and redder peak wavelength than the $H_{*}=0.9 \times 10^{11} \mathrm{~cm}$ solution, reversing the trend of the other solutions. This points to a more serious problem, which was discussed in Paper I: in the low- $H_{*}$ solutions, much of the boundary layer is located at $R<R_{*}$. This can be seen by 
noting that $d \Omega / d R$ is still quite large at $R_{*}$, so that $\Omega$ must continue to drop inside $R_{*}$. Some portion of the boundary layer flux is thus not being included in the spectrum.

In order to avoid these problems, we need to specify an additional boundary condition. The need for an additional condition is also clear from the fact that we have needed to specify values for two of the three variables $\Omega_{*}, H_{*}$, and $j$. In nature, one expects that the stellar rotation rate $\Omega_{*}$ is the only true variable, and that the disk height at the stellar surface $H_{*}$ and the angular momentum accretion rate $j$ will be determined by the accretion flow. An additional condition will allow us to specify only $\Omega_{*}$ in our solutions, and the values of $H_{*}$ and $j$ will follow. As discussed in Paper I, we have selected a somewhat arbitrary condition on the radial velocity at $R_{*}, v_{R * *}=-1000 \mathrm{~cm} \mathrm{~s}^{-1}$. In practice, this keeps the value of $H_{*}$ nearly constant with varying $\Omega_{*}$. As we showed in Paper I, $j$ drops fairly rapidly as $\Omega_{*}$ increases, and becomes negative when $\Omega_{*}$ reaches 20 to $40 \%$ of the breakup stellar rotation rate $\Omega_{K}\left(R_{*}\right)$.

With this additional condition, we are ready to examine spectra and line profiles for low- $j$ solutions. Figure 4 shows five solutions with different values of $j=0.94,0.9,0.84$, 0.6 , 0 , which lie along the track with $v_{R, *}=-1000 \mathrm{~cm} \mathrm{~s}^{-1}$. The stellar rotation rate $\Omega_{*}$ increases as $j$ drops; $j=0$ at $\Omega_{*}=1.55 \times 10^{-5} \mathrm{~s}^{-1} \simeq 0.3 \Omega_{K}\left(R_{*}\right)$. The high- $j$ solutions have $\Omega$ reaching a maximum and then dropping down to $\Omega_{*}$, while in the low- $j$ solutions, $\Omega$ continues to increase all the way in to $R_{*}$. The high- $j$ solutions also have a more pronounced peak in $T_{\text {eff }}$, while the low- $j$ solutions have lower effective temperatures in the boundary layer, but higher in the disk. The high- $j$ solutions produce centrally-peaked line profiles similar to the ones in Figs. 1 and 3. On the other hand, the $j=0.6$ and $j=0$ solutions produce double-peaked profiles. The low $j$ solutions also have redder spectra. The $j=0$ spectrum is more luminous than the others, but it peaks in the red, and drops off rapidly in the blue and ultraviolet due to the lack of a high- $T_{\text {eff }}$ boundary layer region. Thus, we find that low- $j$ solutions match the observations better than high- $j$ solutions. None of the high- $j$ solutions have spectra and line profiles which agree with observations; they either produce centrally-peaked line profiles, or spectra which are bluer than the observed ones. The $j=0$ solution, with moderate values of both $\Omega_{*}$ and $H_{*}$, produces spectra and line profiles which are in much better qualitative agreement with the observations. Note that this conclusion is based on solutions calculated with $\alpha=0.01$; we discuss solutions for other values of $\alpha$ below.

Why do low- $j$ solutions fit the observations better? Some insight into this can be gained by noting that $\mathrm{KHH}$ were able to find good agreement with spectra and line profiles of the same systems, using standard thin disk models with the usual run of effective temperatures and rotational velocities. This leads one to wonder why both types of disk models, with 
or without boundary layers, can be successful in reproducing the observed characteristics of FU Orionis systems. The answer lies in the fact that the boundary layer solutions which best fit the observations, our low- $j$ solutions, in some sense lack a boundary layer. Conversely, more conventional high- $j$ solutions clearly do not agree with the observations.

The low- $j$ solutions do not show the rapid drop in the angular velocity between the disk and the star which is usually associated with the boundary layer region. Instead, $\Omega$ increases slowly inward, so that the rotational velocity in the innermost region of the disk stays nearly constant over a wide range of radius. This produces double-peaked line profiles like the ones observed in FU Orionis systems. Also, $\Omega_{*}$ is moderately large, generally around $20 \%$ of the breakup stellar rotation rate $\Omega_{K}\left(R_{*}\right)$, so that there is no part of the inner disk which has very low rotational velocity. This inner portion of the disk produces most of the optical flux, so any region of it which has very low rotational velocity tends to produce a peak in the center of the line profile, eliminating the double-peaked structure.

A second important characteristic of the low- $j$ solutions is that they lack the pronounced peak in the effective temperature which is characteristic of conventional high- $j$ boundary layer solutions. In high- $j$ solutions, this peak is produced by the rapid release of energy which accompanies the rapid drop in $\Omega$. This energy is released at fairly high effective temperatures, which produces blue and ultraviolet fluxes substantially in excess of those observed. For example, the high- $j$, low- $H_{*}$ solutions in Fig. 3 reach effective temperatures of $\sim 10,000 \mathrm{~K}$, whereas low- $j$ solutions for the same parameters only reach about $7,500 \mathrm{~K}$. At the same time, low- $j$ solutions are fairly luminous, as illustrated by Fig. 4. Although these solutions are cooler than high- $j$ solutions in the innermost region, they are hotter in the remainder of the disk. This is a result of the different temperature distribution of a disk with a different value of $j$. For example, if we adopt the standard thin disk assumptions that $\Omega=\Omega_{K}$ and that the flux radiated from the disk surface is just the energy per unit area released by viscous dissipation, then the effective temperature of the disk is given by

$$
T_{e f f}=\left(\frac{3 G \dot{M} M_{*}}{8 \pi \sigma R^{3}}\right)^{1 / 4}\left[1-j\left(\frac{R_{*}}{R}\right)^{1 / 2}\right]^{1 / 4} .
$$

For $j=1$, we recover the usual expression for $T_{e f f}$, but for $j=0$, the factor in square brackets is eliminated, producing higher effective temperatures. Note that this expression only applies when the thin disk assumptions listed above are satisfied, so that it will not apply to the innermost portion of the disk, where $\Omega$ deviates strongly from Keplerian, and radial energy transport is important. However, at larger radii, the disk comes closer to satisfying these conditions, and $j=0$ solutions should produce higher effective temperatures than high- $j$ solutions. This is confirmed by Fig. 3, where the $j=0$ solution is cooler than the high- $j$ solutions at $R=R_{*}$, but hotter at $R=3 R_{*}$. 
Solutions with $j \simeq 0$ are also "equilibrium" solutions which allow the star to accrete without spinning up or down (see Paper I and $\S 5$ for further discussion of this point). For these reasons, we concentrate on $j=0$ solutions in the remainder of this paper.

\subsection{Viscosity Parameter $\alpha$}

One additional parameter that must be specified in our model is the viscosity parameter $\alpha$. Most estimates of $\alpha$ are based largely on the assumption that the outbursts observed in dwarf novae proceed on the viscous timescale. This produces fairly large values of $\alpha \sim 0.1-1$; however, application of the same idea to FU Orionis outbursts suggests that much smaller values of $\alpha$ are required to reproduce the long outbursts observed in these systems. Bell \& Lin (1994) found that disk instability models for FU Orionis systems required values of $\alpha \sim 10^{-4}$ where hydrogen is neutral and $\alpha \sim 10^{-3}$ where hydrogen is ionized. However, such small values of $\alpha$ lead to problems with evolutionary timescales and gravitational instability in the disk (Bell et al. 1995).

In this work, we have adopted a compromise value of $\alpha=10^{-2}$ for most of our models. In order to explore the effects of varying $\alpha$ in our models, we have calculated a sequence of solutions with $\log \alpha=-1.0,-1.5,-2.0,-2.5,-3.0$. These solutions all have the same values of $\dot{M}=10^{-4.15} \mathrm{M}_{\odot} \mathrm{yr}^{-1}, M_{*}=0.5 \mathrm{M}_{\odot}, R_{*}=3 \times 10^{11} \mathrm{~cm}$, and $j=0$, and we have varied the value of $v_{R, *}$ such that $v_{R, *}=-1000(\alpha / 0.01) \mathrm{cm} \mathrm{s}^{-1}$. These solutions are shown in Figure 5.

The larger values of $\alpha$ correspond to thinner disks, which produce faster stellar rotation rates and higher effective temperatures in the inner disk. If we were to continue to increase $\alpha$ (perhaps to unreasonably large values $\alpha>1$ ), eventually we would reach a nearly Keplerian, thin disk solution. Such a solution would have the star rotating near breakup, and would resemble the solutions found by KHH. The smallest value, $\alpha=10^{-3}$, produces a solution in which the rotation rate is very small throughout the inner disk. This results in a very narrow, centrally-peaked line profile. Larger values of $\alpha$ produce progressively wider double-peaked profiles. There is also a clear trend in the spectra as $\alpha$ changes; larger values of $\alpha$ produce bluer spectra and higher total luminosities. The variation in luminosity is due to the advection of a larger fraction of the accretion luminosity into the star in the small- $\alpha$ solutions, where the disk has become quite thick. 


\section{Comparison to Observed Systems}

We now use our disk and boundary layer solutions to fit the spectra and line profiles of the two best-observed FU Orionis systems: FU Orionis and V1057 Cygni. Both have resolved line profiles available in both the optical and near-infrared, along with broad-band photometry which illustrates the shape of the spectral energy distribution from $3600 \AA$ out to $10 \mu \mathrm{m}$ and beyond. These were two of the original three systems discussed by Herbig (1977) in his seminal paper. The third system, V1515 Cygni, has very narrow, unresolved line profiles which suggest that it is probably viewed pole-on, making it difficult to constrain models for this system. Another well-observed system which is a probable member of the FU Orionis class is Z CMa, which has very broad line profiles. Unfortunately, this system has a binary companion which greatly alters the shape of the infrared spectrum. The other candidate systems have some of these data available, but not all of them, and for many of them their identification as FU Orionis systems is still somewhat uncertain (see Hartmann \& Kenyon 1996 for a discussion of these objects).

\subsection{Observations}

Most of the observations of FU Orionis and V1057 Cygni used in this work are the ones which were used by KHH in their comparison to Keplerian thin disk models. These consist of high-resolution spectra, which are used to derive line profiles, and photometry to show the broad-band spectral energy distributions of these objects. The high-resolution spectra

include echelle spectra of a 50- $\AA$ region around $6170 \AA$, and Fourier transform spectra of the $2.2 \mu \mathrm{m}$ region. We have used a more recent $2.2 \mu \mathrm{m}$ spectrum of V1057 Cyg with improved resolution of $\sim 10 \mathrm{~km} \mathrm{~s}^{-1}$. Unlike the earlier spectrum, the cross-correlation produced from this spectrum is clearly double-peaked. As described by KHH, the photometric data include low-resolution optical spectrophotometry which has been binned into the standard photometric bands, along with standard optical and infrared photometry. The photometric data have been dereddened using the extinction law given by Savage \& Mathis (1979) and assuming $A_{V}=2.2 \mathrm{mag}$ for FU Orionis and $A_{V}=3.5 \mathrm{mag}$ for V1057 Cygni.

\section{2. $\quad$ Fitting Procedure}

Based on the results discussed in $\S 3$, in attempting to fit the observations, we have fixed certain parameters: since low- $j$ solutions seem to fit the general characteristics of the observations, we have fixed $j=0$. We also have fixed the boundary condition on 
$v_{R, *}=-1000 \mathrm{~cm} \mathrm{~s}^{-1}$. This together with the condition on $j$ fixes the position of the solution in the $\Omega_{*}-H_{*}$ plane (see Fig. 2). We also fix the value of $\alpha$ at $10^{-2}$. This leaves the standard accretion parameters $\dot{M}, M_{*}$, and $R_{*}$, which we vary in order to fit the observations. As discussed in $\S 3.1$, we can use these three parameters to calculate $L_{a c c}=G M_{*} \dot{M} / R_{*}, T_{\max }=0.287\left(G M_{*} \dot{M} / \sigma R_{*}^{3}\right)^{1 / 4}$, and $v_{K}\left(R_{*}\right)=\left(G M_{*} / R_{*}\right)^{1 / 2}$, which relate more directly to the observations which we are trying to match. These parameters give us a good sense of how changes in $\dot{M}, M_{*}$, and $R_{*}$ will affect our spectra and line profiles. For instance, if we want to increase the luminosity of a particular solution without changing the temperature or rotational velocity, the dependences given above suggest that we need to keep the ratios $\dot{M} M_{*} / R_{*}^{3}$ and $M_{*} / R_{*}$ constant. If we increase $R_{*}$, we can increase $M_{*}$ by the same factor and increase $\dot{M}$ as $R_{*}^{2}$. This will increase $L_{a c c}$ as $R_{*}^{2}$ while keeping $T_{\max }$ and $v_{K}\left(R_{*}\right)$ constant.

There are several other parameters which enter into our fits: the inclination angle, distance, and extinction of the individual FU Orionis systems. The inclination angle $i$ and the distance $d$ affect the derived luminosity of the FU Orionis systems, since we convert the luminosity of our solutions to flux using the relation $F_{\lambda}=L_{\lambda} \cos i / 2 \pi d^{2}$, and then compare these fluxes to the observed ones. The inclination angle also affects the linewidths derived from our solutions, since the linewidth is set by the line-of-sight velocity of the disk material $v_{\text {los }}=v_{\text {rot }} \sin i$. The extinction $A_{V}$ affects both the shape and the luminosity of the spectra, since the observed photometric points are dereddened before they are used for comparison with the solutions. We adopt values published elsewhere for the distance (Hartmann \& Kenyon 1996) and extinction (KHH), and leave these fixed. We vary the inclination as part of our fitting procedure.

Our fits to the observations are all approximate, and are done by eye. In comparing the spectra produced by our solutions with photometric data, we weight some of the data points less heavily than others. At wavelengths longer than $10 \mu \mathrm{m}$, the data frequently show that the objects are much brighter than our models would predict. This is generally attributed to the presence of reprocessing of some of the disk luminosity by the outer disk or by a dusty envelope surrounding the disk (KHH; Kenyon \& Hartmann 1991). Thus we confine our comparison with the photometric data to wavelengths shorter than $10 \mu \mathrm{m}$. At the blue end of the spectrum, the U-band point at $3600 \AA$ is generally substantially fainter than the B-band point at $4400 \AA$. This rapid drop in the spectral energy distribution is probably due largely to the Balmer jump, and it is therefore difficult to reproduce it with a blackbody spectrum. Our spectra produced using a library of stellar spectra tend to be closer to the observed point. A number of other factors could also contribute to the rapid dropoff from $\mathrm{B}$ to $\mathrm{U}$; the extinction increases dramatically as one moves to shorter wavelengths in this portion of the spectrum, and so error in the dereddening applied to 
the observed spectrum will have their greatest effect here. Also, inclination effects tend to depress the blue end of the spectrum.

When we compare our line profiles to the data, we do not compare them directly to individual line profiles, which tend to be noisy and frequently blended with other nearby lines, but rather to the shape of the cross-correlation function. The cross-correlation function represents the mean line shape in the spectral region for which it is derived. We have assigned the zero point of our model line profiles to the zero point of the cross-correlations, and normalized the two by their peak heights. We quantitatively compare the overall shape of our line profile to the cross-correlation by determining the velocity widths of the two at half-maximum and at full-maximum. We also compare the ratio of the optical and infrared line widths produced by our solutions to the ratio seen in the data.

\subsection{Fits to Individual Systems}

\subsubsection{FU Orionis}

This object has broad line profiles, with full-width at half-maximum of $100 \mathrm{~km} \mathrm{~s}^{-1}$ at $6170 \AA$ and $74 \mathrm{~km} \mathrm{~s}^{-1}$ at $2.2 \mu \mathrm{m}$. It is also quite bright, with a peak value of $\log \lambda F_{\lambda} \simeq-7.5$ in the $\mathrm{V}$-band, assuming $A_{V}=2.2$. Adopting a distance of $500 \mathrm{pc}$, we find good fits to the line profiles and spectrum from a solution with $\dot{M}=2 \times 10^{-4} \mathrm{M}_{\odot} \mathrm{yr}^{-1}, M_{*}=0.7 \mathrm{M}_{\odot}$, $R_{*}=4 \times 10^{11} \mathrm{~cm}$, and $i=60^{\circ}$. As mentioned above, the solution has $j=0$ and $\alpha=0.01$. It also has $v_{R, *}=-1000 \mathrm{~cm} \mathrm{~s}^{-1}$, giving a stellar rotation rate of $8.29 \times 10^{-6} \mathrm{~s}^{-1}$ and a disk height at the stellar radius $H_{*}=1.57 \times 10^{11} \mathrm{~cm}$. These values correspond to a stellar rotation period of 8.76 days and $H_{*} / R_{*}=0.39$.

The angular velocity $\Omega$ and rotational velocity $v_{\text {rot }}=\Omega R$ are shown in Figure 6 a. The rotational velocity at the stellar surface is only $33 \mathrm{~km} \mathrm{~s}^{-1}$, and the maximum rotational velocity is $47 \mathrm{~km} \mathrm{~s}^{-1}$ at $R \simeq 8.75 \times 10^{11} \mathrm{~cm} \simeq 2.19 R_{*}$. Figure $6 \mathrm{~b}$ shows the effective temperature $T_{e f f}$, which reaches a maximum value of $8212 \mathrm{~K}$ at $R \simeq 4.6 \times 10^{11} \mathrm{~cm}=1.15 R_{*}$. Figure 6c shows the line profiles derived from this disk model at $6170 \AA$ and $2.2 \mu \mathrm{m}$, respectively. Both profiles are double-peaked, like the cross-correlations, and we find FWHM values of 98 and $72 \mathrm{~km} \mathrm{~s}^{-1}$ at the two wavelengths. Note that the cross-correlation peaks are rather asymmetric; this is probably due to the presence of a mass loss in a wind, as discussed by KHH. Figure 6d shows the fits of blackbody and stellar composite spectra to the photometry of FU Orionis. Both spectra fit the data points quite well from the B band to $10 \mu \mathrm{m}$, deviating from most data points by $10 \%$ or less. Both are too bright in the $\mathrm{U}$ band, although the stellar composite spectrum comes substantially 
closer to the observed point. The luminosity emitted by the boundary layer and disk is $L \simeq 1.83 \times 10^{36} \operatorname{ergs~s}^{-1} \simeq 470 \mathrm{~L}_{\odot}$.

If this were a standard Keplerian thin disk, the disk parameters $\dot{M}, M_{*}$, and $R_{*}$ for this solution would give an accretion luminosity $L_{a c c} \simeq 2.95 \times 10^{36} \mathrm{ergs} \mathrm{s}^{-1} \simeq 750 \mathrm{~L}_{\odot}$, a maximum disk temperature $T_{\max } \simeq 6850 \mathrm{~K}$, and a Keplerian rotational velocity

$v_{K}\left(R_{*}\right) \simeq 150 \mathrm{~km} \mathrm{~s}^{-1}$. These are substantially different from the actual luminosity, peak effective temperature, and peak rotational velocity found in our solution. These differences indicate the importance of including the boundary layer region in our disk model.

\subsubsection{V1057 Cygni}

This system has narrower line profiles than FU Orionis, with FWHM values of $57 \mathrm{~km} \mathrm{~s}^{-1}$ at $6170 \AA$ and $47 \mathrm{~km} \mathrm{~s}^{-1}$ at $2.2 \mu \mathrm{m}$. It also appears to be somewhat fainter; after dereddening with $A_{V}=3.5$, it has a peak $\log \lambda F_{\lambda} \simeq-7.8$, and the peak occurs in the $\mathrm{R}$ band, indicating that this system is slightly cooler than FU Orionis. We adopt a distance of 600 pc.

We find good fits to the spectrum and line profiles of V1057 Cygni from a disk and boundary layer solution with $\dot{M}=10^{-4} \mathrm{M}_{\odot} \mathrm{yr}^{-1}, M_{*}=0.5 \mathrm{M}_{\odot}$, and $R_{*}=3.5 \times 10^{11} \mathrm{~cm}$. This solution is less luminous and slightly cooler than the solution for FU Orionis described above, but in most other respects the two solutions are quite similar. Like the solution for FU Orionis, this solution has $j=0, \alpha=10^{-2}$, and $v_{R, *}=-1000 \mathrm{~cm} \mathrm{~s}^{-1}$. This gives a stellar rotation rate $\Omega_{*}=8.88 \times 10^{-6} \mathrm{~s}^{-1}$, which corresponds to a rotation period of 8.19 days. The disk height at $R_{*}$ is $H_{*}=1.37 \times 10^{11} \mathrm{~cm}$, so $H_{*} / R_{*}=0.39$.

The angular and rotational velocities of this solution are shown in Figure 7a. The rotational velocity is $31 \mathrm{~km} \mathrm{~s}^{-1}$ at the stellar surface, and reaches a peak value of $49 \mathrm{~km} \mathrm{~s}^{-1}$ at $R=8.49 \times 10^{11} \mathrm{~cm}=2.43 R_{*}$. The effective temperature, shown in Figure $7 \mathrm{~b}$, reaches a maximum value of $7111 \mathrm{~K}$ at $R \simeq 4 \times 10^{11} \mathrm{~cm}=1.14 R_{*}$. Figure $7 \mathrm{c}$ shows the line profiles at $6170 \AA$ and $2.2 \mu \mathrm{m}$ for this solution. The profiles have FWHM values of 54 and $44 \mathrm{~km} \mathrm{~s}^{-1}$, respectively, compared to the observed values of 57 and $47 \mathrm{~km} \mathrm{~s}^{-1}$. The ratio of the optical to infrared linewidth is quite close to the observed ratio, where the KHH thin disk model gave infrared linewidths which were about $25 \%$ smaller than those observed. The separation of the two peaks is 26 and $14 \mathrm{~km} \mathrm{~s}^{-1}$ in our disk solution, and 33 and $19 \mathrm{~km} \mathrm{~s}^{-1}$ in the data. The fits of our blackbody and stellar composite spectra to the photometric data for V1057 Cygni are shown in Figure 7d. The blackbody spectrum fits quite well in both the optical and near-infrared regions; it is too bright in the U-band, as expected. The data 
show a drop in the I-band and an excess at 3.5 and $4.8 \mu \mathrm{m}$; these points would be difficult to fit with any smooth curve which also fits the optical and near-infrared points. The stellar composite spectrum comes much closer to the U-band point but is slightly fainter than the data in the optical.

\subsection{Comparison to Thin Disk Models}

It is interesting to compare the disk parameters $\dot{M}, M_{*}$, and $R_{*}$ of our best-fit models for FU Orionis and V1057 Cygni to those found by KHH using thin disk models. KHH list $M_{*}$ and $R_{*}$ for each system as a function of the inclination angle $i$; these values of $M_{*}$ and $R_{*}$ keep the projected rotational velocity at the stellar surface $\left(G M_{*} / R_{*}\right)^{1 / 2} \sin i$ constant at $93 \mathrm{~km} \mathrm{~s}^{-1}$ for FU Orionis and $42.6 \mathrm{~km} \mathrm{~s}^{-1}$ for V1057 Cygni. KHH also keep the maximum disk temperature constant at $7200 \mathrm{~K}$ in FU Orionis and $6590 \mathrm{~K}$ in V1057 Cygni, so $\dot{M}$ also varies with inclination. Our models use an inclination of $60^{\circ}$ for FU Orionis and $30^{\circ}$ for V1057 Cygni. For these inclinations, KHH found $M_{*}=0.34 \mathrm{M}_{\odot}, R_{*}=5.47 \mathrm{R}_{\odot}$, and $\dot{M}=4.36 \times 10^{-4} \mathrm{M}_{\odot} \mathrm{yr}^{-1}$ for $\mathrm{FU}$ Orionis, and $M_{*}=0.15 \mathrm{M}_{\odot}, R_{*}=4.02 \mathrm{R}_{\odot}$, and $\dot{M}=2.75 \times 10^{-4} \mathrm{M}_{\odot} \mathrm{yr}^{-1}$ for V1057 Cygni.

Our best-fit models give $M_{*}=0.7 \mathrm{M}_{\odot}, R_{*}=5.75 \mathrm{R}_{\odot}$, and $\dot{M}=2 \times 10^{-4} \mathrm{M}_{\odot} \mathrm{yr}^{-1}$ for FU Orionis, and $M_{*}=0.5 \mathrm{M}_{\odot}, R_{*}=5.03 \mathrm{R}_{\odot}$, and $\dot{M}=10^{-4} \mathrm{M}_{\odot} \mathrm{yr}^{-1}$ for $\mathrm{V} 1057$ Cygni. Thus we find stellar masses which are a factor of $2-3$ larger, mass accretion rates which are a factor of 2-3 smaller, and slightly larger stellar radii. These differences are due to the fact that our models include the boundary layer region. Thus, rotational velocities are substantially below Keplerian in the inner disk, requiring larger stellar masses to produce the observed linewidths. To compensate for this increased stellar mass, the mass accretion rate must decrease by a similar factor in order to produce similar luminosities and temperatures. Note that the KHH models only included the disk luminosity, which is half of the total accretion luminosity in the standard thin disk model. Our models include both the disk and boundary layer luminosities, but they do not radiate the full accretion luminosity, as mentioned in $\S 4.3$ and discussed below. Finally, the presence of the boundary layer increases the inner disk temperatures, and so a slightly larger stellar radius is required to keep the spectra from becoming too blue.

\section{Discussion}




\subsection{Standard Disk Solutions vs. Disk and Boundary Layer Solutions}

We have found disk and boundary layer solutions with significant departures from Keplerian rotation that can reproduce the key spectral features of FU Orionis and V1057 Cygni. However, observations of these objects were originally modelled with reasonable success using standard Keplerian disk theory (Hartmann \& Kenyon 1985; KHH). Here we discuss why it is difficult to compare the relative merits of the two types of solutions on the basis of observations alone. We then outline why we think the boundary layer solutions are nevertheless an important improvement in treating FU Orionis accretion physics.

Both standard thin disk solutions and disk and boundary layer solutions agree quite well with observations. Thus, comparisons with observations do not provide a definitive test of the relative merits of the two types of solutions for FU Orionis objects. The disk and boundary layer solutions appear to match the differential rotation with wavelength somewhat better than the thin disk solutions of KHH; the thermal pressure support in the inner regions of the disk tends to reduce the ratio of the optical line widths to the near-infrared line widths, as observed. However, as pointed out by KHH, there are significant uncertainties in strengths of the infrared CO lines in the outer disk due to the possibility of dust formation, and with plausible models of dust contributions a thin disk in Keplerian rotation can match the observed differential rotation with wavelength.

Similarly, differences in how well the two types of solutions match the observed spectral energy distributions, or small differences in the inferred masses, radii, and accretion rates, are not significant in view of the many uncertainties involved. Specifically, there are uncertainties in the inclination angles $i$, distances $d$, and extinctions $A_{V}$ of FU Orionis and V1057 Cygni. We have used fairly standard inclinations of $i=60^{\circ}$ for $\mathrm{FU}$ Orionis and $i=30^{\circ}$ for V1057 Cygni. These inclinations allow solutions with similar rotational velocities to produce the rather different linewidths seen in these two systems. In fact, the ratio $\sin 30^{\circ} / \sin 60^{\circ} \simeq 0.58$ is almost identical to the ratio of the $6170 \AA$ linewidths (57 and $100 \mathrm{~km} \mathrm{~s}^{-1}$ ) in these objects. Nonetheless, other inclinations, in combination with different choices of the other solution parameters, might produce solutions which match the data. The distances to FU Orionis and V1057 Cygni are probably only accurate to 10-20\%, so that the luminosities inferred for these systems could be in error by 20-40\%. Finally, the extinctions of these systems are not well known, and could represent an additional source of error in both the luminosities and the shape of the spectra. All of these considerations limit our ability to distinguish between different disk models.

Nonetheless, we feel that the disk and boundary layer model presented in this paper offers significant advantages over the standard thin disk model. First, it avoids some uncomfortable assumptions which are implicit in the thin disk model. The two major 
observational constraints on models of the inner disk in FU Orionis systems are the apparent lack of hot boundary layer emission and the lack of slowly-rotating material in the observed line profiles. The absence of these features gives the impression that the boundary layer is not present is FU Orionis systems. The standard thin disk model (Hartmann \& Kenyon 1985; KHH) simply did not include any boundary layer region. By omitting the boundary layer, this model made the implicit assumption that the accreting star is rotating at breakup speed. Rapid stellar rotation seems unlikely in view of the generally slow rotations of T Tauri stars, plus the recognition that FU Orionis and V1057 Cygni have not accreted enough angular momentum during their outbursts to spin up the entire central star as a solid body (Hartmann \& Kenyon 1996). This leaves open the possibility that only the outer layers are spun up to Keplerian angular velocities. A second implicit assumption of the early model is that the disk can be Keplerian all the way in to the stellar surface, which implies that pressure gradients in the inner disk are insignificant. This also seems unlikely; at the high mass accretion rates found in FU Orionis systems, radial pressure gradients will support the accreting material and produce sub-Keplerian angular velocities unless $\alpha$ is very large.

Our disk and boundary layer model avoids these assumptions. We have found that by using the slim disk model, we can include the boundary layer region in our calculations of FU Orionis disks and still find solutions which agree with the observations. These solutions demonstrate explicitly what the thin disk model implicitly assumed: FU Orionis disks do not have a standard accretion disk boundary layer where the angular velocity of the accreting material drops rapidly over a short radial distance. Unless $\alpha$ is very large, the high accretion rates of FU Orionis objects require quite optically thick disks which are very hot in their interiors, resulting in signficant thermal pressure support. Thus the narrow, hot boundary layer typically expected in pre-main-sequence stars (Lynden-Bell \& Pringle 1974) automatically disappears, for a wide range of stellar rotation rates. Instead, the "boundary layer" is a broad region where $\Omega$ changes gradually; in our best-fitting solutions, $\Omega$ increases gradually and becomes nearly constant as the accreting material approaches the star. As a result, like the early thin disk solutions, our solutions lack the high-temperature, slowly-rotating region expected from a standard boundary layer. But unlike the early solutions, they do not require that the star be rotating at breakup or that $\alpha$ be very large. In fact, for $\alpha=10^{-2}$, our best solutions have the star rotating at only $\sim 20-25 \%$ of breakup speed. Fig. 5 shows that as $\alpha$ increases, $\Omega_{*}$ increases, reaching $\sim 50 \%$ of breakup speed at $\alpha=0.1$. This suggests that the Keplerian thin disk solutions of Hartmann \& Kenyon (1985) and KHH represent a special, extreme case of the solutions found in this paper, where the values of both $\alpha$ and $\Omega_{*}$ are very large.

In addition to eliminating the assumptions of very large values of $\alpha$ and $\Omega_{*}$ which are 
implicit in the thin disk model, our disk and boundary layer model can offer insight into new areas of FU Orionis accretion physics. The model explicitly includes the effects of the rotation rate of the star on the boundary layer region and on the angular momentum accretion rate. Since radial advection of energy is included in the slim disk equations, our solutions directly give the rate at which energy is carried into the accreting star. These issues can only be studied when the boundary layer region and the additional physics of the slim disk equations are included in the model. They have important consequences for the evolution of pre-main-sequence stars, which will be discussed in more detail in the following sections.

\subsection{Low- $j$ Solutions: Implications for Spin Evolution of Pre-Main-Sequence Stars}

Our best-fit solutions for FU Orionis and V1057 Cygni are equilibrium solutions with

$j=0$, i.e. the central star is neither gaining nor losing angular momentum, and have stellar rotation rates $\Omega_{*} \simeq 0.225 \Omega_{K}\left(R_{*}\right)$ which correspond to rotation periods of 8-9 days. These periods are comparable to those observed in T Tauri stars (Bouvier et al. 1993, 1995). These low- $j$ solutions have important implications for the spin evolution of pre-main-sequence stars, because they support the scenario proposed in Paper I, where FU Orionis outbursts regulate the rotation rates of $\mathrm{T}$ Tauri stars. In Paper I we showed that the angular momentum accretion rate $j$ drops rapidly as the stellar rotation rate $\Omega_{*}$ increases, and reaches $j=0$ when $\Omega_{*} \simeq 0.2-0.4 \Omega_{K}\left(R_{*}\right)$ for FU Orionis parameters. For smaller values of $\Omega_{*}, j>0$ and the star spins up, while for larger values of $\Omega_{*}, j<0$ and the star spins down. Thus, FU Orionis outbursts will move $\Omega_{*}$ toward an equilibrium value where $j \simeq 0$. If FU Orionis outbursts dominate mass accretion onto T Tauri stars, as seems likely from event statistics, then they should also dominate angular momentum accretion and control the spin evolution of these stars. When the T Tauri star is between outbursts, it may spin up or down, but during each outburst it will return to the equilibrium rotation rate. This scenario therefore predicts that FU Orionis systems should attain a low- $j$ equilibrium state during outbursts, and we find that low- $j$ solutions fit the observations.

We are unable to make more precise predictions of the spin rates of $\mathrm{T}$ Tauri stars because of uncertainties in the structure of the central star undergoing FU Orionis accretion. As discussed in $§ 5.4$ below, it is likely that the advection of large amounts of thermal energy through the inner disk will cause the central star to expand from its equilibrium state. After an outburst ceases, we expect that the star will contract to a smaller radius, and will therefore spin up somewhat in the absence of any angular momentum loss. Note that the 
disk may still remove large amounts of angular momentum from the star and spin the star down substantially during the course of the outburst; however, at the end of the outburst, we expect that there will always be some spinup due to the contraction of the star. Thus the equilibrium rotation rates during the FU Orionis phase should correspond to faster $\mathrm{T}$

Tauri rotation rates. The amount of spinup will depend upon the moment of inertia of the expanded layers of the star, which we are not able to estimate at present.

Observations of T Tauri stars suggest that classical T Tauri stars which have accretion disks rotate more slowly than weak-line T Tauri stars which lack disks (Bouvier et al. 1993, 1995; Edwards et al. 1993; Eaton, Herbst, \& Hillenbrand 1995; Choi \& Herbst 1996). Our results, combined with those of Paper I, suggest a new way in which the disk can regulate the rotation rate of an T Tauri star. Previously suggested methods of regulating the stellar rotation rate have relied upon the interaction between the stellar magnetic field and the disk (Königl 1991; Cameron \& Campbell 1993; Hartmann 1994; Shu et al. 1994). Our method does not depend on the stellar magnetic field, which is presumably not strong enough to disrupt the disk during FU Orionis outbursts. However, it does require that outbursts occur, and that they dominate the angular momentum accretion onto the star.

\subsection{Luminosities}

We noted in $§ 4.3$ that our solutions for FU Orionis and V1057 Cygni produce luminosities which are substantially smaller than the accretion luminosity. For FU Orionis, we found that our solution gave $L=470 \mathrm{~L}_{\odot}$, whereas $L_{a c c} \simeq 750 \mathrm{~L}_{\odot}$, so that $L \simeq 0.63 L_{a c c}$. For our V1057 Cygni solution, $L \simeq 195 \mathrm{~L}_{\odot}$ and $L_{a c c} \simeq 310 \mathrm{~L}_{\odot}$, so we again have $L \simeq 0.63 L_{a c c}$. The remaining luminosity goes into heating the disk material. At the stellar surface, the midplane temperature of the disk in our solutions becomes quite large: $T_{c}\left(R_{*}\right) \simeq 2.5 \times 10^{5} \mathrm{~K}$ in the FU Orionis solution, and $2 \times 10^{5} \mathrm{~K}$ in the V1057 Cygni solution. This means that the disk material carries energy into the star at the rate $\dot{M} c_{P} T_{c}\left(R_{*}\right) \simeq 280 \mathrm{~L}_{\odot}$ for $\mathrm{FU}$ Orionis and $115 \mathrm{~L}_{\odot}$ for $\mathrm{V} 1057$ Cygni. The high temperature of the disk material is due to the large vertical optical depth of the disk and boundary layer in these solutions. If the accreting stars in FU Orionis objects are typical pre-main-sequence stars, they should have luminosities $\sim 1-10 \mathrm{~L}_{\odot}$, so this advected energy represents a major perturbation to the star.

Another interesting feature of these solutions is that only a fraction of the luminosity comes from energy liberated by viscous dissipation. Popham \& Narayan (1995) derived an 
expression for the total viscous dissipation rate of the disk and boundary layer

$$
L_{\text {diss }}=\frac{G M_{*} \dot{M}}{R_{*}}\left(1-j \frac{\Omega_{*}}{\Omega_{K}\left(R_{*}\right)}+\frac{1}{2} \frac{\Omega_{*}^{2}}{\Omega_{K}^{2}\left(R_{*}\right)}\right)+\dot{M} \int \frac{d P}{\rho},
$$

where we have left out several small terms. The first term in this expression represents the gravitational potential energy and the rotational kinetic energy transferred between the star and the disk. For $j=0$, the disk is removing a small amount of rotational kinetic energy from the star. Both of our solutions have $\Omega_{*} / \Omega_{K}\left(R_{*}\right)=0.225$, so the first term is just $1.025 L_{a c c}$. In a thin disk, the second term is very small, but in our FU Orionis solutions, this term becomes quite large due to the importance of the radial pressure gradient in supporting the accreting material against gravity. In both solutions, we find $\dot{M} \int d P / \rho=-0.74 L_{a c c}$, so that the viscous dissipation rate is only $L_{d i s s}=0.285 L_{a c c}$. Nonetheless, we know that both solutions radiate $L \simeq 0.63 L_{a c c}$; the remaining $\sim 0.35 L_{a c c}$ comes from the entropy term in the energy equation

$$
\nu \Sigma\left(R \frac{d \Omega}{d R}\right)^{2}-F_{V}-\Sigma v_{R} T_{c} \frac{d S}{d R}-\frac{1}{R} \frac{d}{d R}\left(R H F_{R}\right)=0 .
$$

The four terms in this equation represent the viscous dissipation, the vertical flux from the disk surface, the advected entropy, and the divergence of the radial flux. If we integrate this equation over the surface of the disk, we have

$$
L_{\text {diss }}-L+\dot{M} \int T d S-4 \pi\left[R_{\text {out }} H_{\text {out }} F_{R, \text { out }}-R_{*} H_{*} F_{R, *}\right]=0 .
$$

Our boundary conditions assume that the radial fluxes at the inner and outer edges of the disk are small; at the inner edge we assume that the flux entering the disk is $\sigma T_{*}^{4}$, where we assume $T_{*}=5000 \mathrm{~K}$, so $4 \pi R_{*} H_{*} F_{R, *} \sim 0.01-0.02 L_{a c c}$, and at the outer edge the radial flux is insignificant. If we neglect these radial flux terms, we can use $T d S=d U-P d \rho / \rho^{2}$ and the expression for $L_{\text {diss }}$ given above to write

$$
\begin{aligned}
& L=L_{a c c}(\left.1-j \frac{\Omega_{*}}{\Omega_{K}\left(R_{*}\right)}+\frac{1}{2} \frac{\Omega_{*}^{2}}{\Omega_{K}^{2}\left(R_{*}\right)}\right)+\dot{M} \int \frac{d P}{\rho}+\dot{M} \int d U-\dot{M} \int \frac{P}{\rho^{2}} d \rho \\
& \simeq L_{a c c}\left(1-j \frac{\Omega_{*}}{\Omega_{K}\left(R_{*}\right)}+\frac{1}{2} \frac{\Omega_{*}^{2}}{\Omega_{K}^{2}\left(R_{*}\right)}\right)+\frac{5}{2} \dot{M} \int d\left(\frac{P}{\rho}\right) \\
& \simeq L_{a c c}\left(1-j \frac{\Omega_{*}}{\Omega_{K}\left(R_{*}\right)}+\frac{1}{2} \frac{\Omega_{*}^{2}}{\Omega_{K}^{2}\left(R_{*}\right)}\right)-\dot{M} c_{P} T_{c}\left(R_{*}\right)
\end{aligned}
$$

where the internal energy $U \simeq 3 P / 2 \rho, c_{P} T_{c} \simeq 5 P / 2 \rho$, and $T_{c}\left(R_{*}\right) \gg T_{c}\left(R_{\text {out }}\right)$. This accounts for the difference between the actual luminosities radiated by our solutions and the accretion luminosities for those disk parameters. 


\subsection{Stellar Radii and Advected Energy}

Our best-fit solutions for FU Orionis and V1057 Cygni have stellar radii of $5-6 \mathrm{R}_{\odot}$. These are substantially larger than the radii of $\mathrm{T}$ Tauri stars, which are generally estimated at $1.5-3 \mathrm{R}_{\odot}$ (Bouvier et al. 1995). Since FU Orionis outbursts are believed to occur in $\mathrm{T}$ Tauri star accretion disks, this seems to imply that the star expands rapidly during the outburst, probably as a result of the rapid addition of high-temperature material (KHH; Hartmann, Cassen, \& Kenyon 1996).

Prialnik \& Livio (1985) calculated the effects of accretion onto a $0.2 \mathrm{M}_{\odot}, 0.2 \mathrm{R}_{\odot}$ fully-convective main-sequence star. They found that if the accretion carries a sufficient amount of energy into the star, it can cause the star to expand stably or unstably. For accretion rates comparable to those of FU Orionis stars, their calculations indicate that the expansion will proceed unstably if the accretion energy is added to the star at a rate faster than about $10 \%$ of the accretion luminosity. The rapid expansion is due to the conversion of the normally convective star to a convectively-stable, radiative structure.

As the discussion of the luminosities shows, our solutions add energy to the accreting star at an enormous rate $-0.375 L_{a c c} \simeq 100-300 \mathrm{~L}_{\odot}$ in our solutions for $\mathrm{FU}$ Orionis and V1057 Cygni. This rate of energy transfer will almost certainly produce rapid expansion of the star. The character of this expansion is not well understood. The accreting material comes from a disk, so it carries angular momentum as well as energy, and is added around the star's equator. Thus the expansion is likely to be nonspherical. More sophisticated models of this process are needed, but the problem is clearly a difficult one. The large energy input into the star and the resulting stellar expansion should have important implications for the decline from FU Orionis outbursts. The star will contract and release the energy it gained during the outburst, which is a substantial fraction of the total accretion energy of the outburst. Thus we predict, qualitatively, that at the end of the mass accretion outburst in the disk, there may be a phase in which the central star is overly luminous, and fades over some (rapid) time set by the Kelvin time of the perturbed portion of the stellar envelope, similar to the original explanation put forth by Larson (1983) for FU Orionis outbursts.

\subsection{Future work}

By including the boundary layer region and the disk structure self-consistently, the solutions presented here represent a substantial advance in our understanding of disks in FU Orionis objects and the spectra and line profiles they produce. Nonetheless, there are additional explorations which could be made within the context of our assumptions, and 
improvements which could be made to our model.

The solutions we have found do a good job of fitting the observed line profiles and spectra of FU Orionis and V1057 Cygni. Nevertheless, this does not mean that they are the only solutions which would fit the observations of these systems. The number of solution parameters is too great to permit a full exploration of the entire parameter space. Accordingly, as described in $\S 4.2$, we have used the standard parameters $L_{a c c}, T_{\max }$, and $v_{K}\left(R_{*}\right)$ to guide our search. We have also kept certain parameters constant in order to simplify the fitting procedure. We keep $j=0$ for the reasons discussed in $\S 3$, where we demonstrated that high- $j$ solutions fail to produce spectra and line profiles that agree with observations. Other values of $j$ could produce reasonable solutions; for instance, in Paper I we proposed that FU Orionis outbursts may spin down the accreting star, which would require solutions with negative values of $j$. Negative- $j$ solutions are similar in all respects to $j=0$ solutions; the boundary layer region has $\Omega$ increasing inward throughout and lacks a strong peak in effective temperature. As we demonstrated in Paper I, $j$ drops rapidly with increasing $\Omega_{*}$, so that a $j=-1$ solution has $\Omega_{*}$ only slightly larger than a $j=0$ solution with the same parameters (see Fig. 2).

We also use $\alpha=10^{-2}$ for all of our solutions. As discussed in $\S 3.3$, solutions with smaller values of $\alpha$ have thicker disks, so that a larger fraction of the accretion luminosity is advected into the star. Thus a smaller fraction is radiated, and the effective temperature is lower. Another consequence of changing $\alpha$ is that the rotational velocities change; small values of $\alpha$ result in small rotational velocities which would produce centrally peaked line profiles unlike those observed. This suggests that values of $\alpha>10^{-2}$ may produce acceptable solutions, but those with $\alpha$ substantially below $10^{-2}$ may not. This is particularly interesting because Bell \& Lin (1994) and Bell et al. (1995) found that they needed $\alpha \sim 10^{-3}-10^{-4}$ to obtain satisfactory outbursts using the thermal instability mechanism.

Our model spectra could also be improved by treating the effects of inclination in more detail. We have implicitly assumed that the disk surface is flat, so that the inclination angle for all parts of the disk surface is the same. In fact, most of our solutions have $H / R$ nearly constant with $R$, with $H / R \sim 0.4$. If we take $H(R)$ as representing the surface of the disk, then the disk surface is inclined at $\tan ^{-1}(H / R) \sim 22^{\circ}$ to the disk midplane. This means that at inclination angles greater than $i \sim 68^{\circ}$, a portion of the disk surface will not be visible. Even if $i<68^{\circ}$, the side of the disk closer to the observer will effectively be seen at a larger inclination angle than the opposite side. (note that when we refer to "sides" of the disk here, we are referring not to the top and bottom surfaces of the disk, but to regions of the same surface at different angles around the rotation axis.) Also, one side of the disk will 
be heated by radiation from the other side. We have also assumed that the disk spectrum does not vary with inclination angle, whereas in fact limb darkening will generally produce a decline in the blue end in the spectrum for systems viewed at large inclination angles.

Finally, there are improvements which could be made in the physical treatment of the disk and boundary layer. The assumption of a steady disk might be relaxed, although we feel this is unlikely to be a major issue, even though we are comparing our solutions to objects experiencing outbursts, because FU Orionis and V1057 Cygni have both remained fairly steady over recent years (KHH; Kenyon \& Hartmann 1991), and we are only addressing the innermost regions of the disk. Time-dependent models of boundary layers in disks around pre-main-sequence stars (Godon 1996) also seem to agree with our steady-state results (PNHK). Calculations of the structure of advection-dominated accretion disks (Narayan \& Yi 1995) have demonstrated that the slim disk equations used in our model provide a fairly good representation of the disk structure even for disks which are quite vertically thick; still, a two-dimensional model for the boundary layer and disk might provide insights into FU Orionis objects that we are unable to make using our current model (e.g., Kley 1991), especially when considering a more subtle matching of the disk to the inherently two-dimensional star. Some consideration should also be given to understanding the effects of rapid accretion on the central star. Presumably these include rapid expansion of the star, which could have important effects on the boundary layer region and the angular momentum accretion by the star. Ultimately this will have to be a two-dimensional, time-dependent calculation as well, but perhaps some progress can be made with calculations similar to those performed by Prialnik \& Livio (1985) for main-sequence convective stars.

\section{Summary}

We have calculated solutions for the structure of the accretion disk in FU Orionis objects which include a self-consistent treatment of the boundary layer region. We have also computed the line profiles and continuum spectra that would be observed from these solutions. We have explored the dependence of these observable characteristics on the solution parameters, including the mass accretion rate, the stellar mass, radius, and rotation rate, the angular momentum accretion rate, and the viscosity parameter. We find that our slim disk solutions can account for the absence of hot boundary layer emission without requiring that the central star rotate at breakup velocity. The differential rotation of the innermost disk departs from Keplerian rotation and gives marginally better agreement with observations than a Keplerian disk, but uncertainties in line formation preclude using the observations to make a definitive test. We find solutions at spin equilibrium $(j \sim 0)$ for 
stellar angular velocities comparable to those observed in T Tauri stars, and these solutions fit the observations of FU Orionis and V1057 Cygni. This result supports the proposal made in Paper I that FU Orionis outbursts play an important role in regulating the rotation rates of $\mathrm{T}$ Tauri stars.

In our solutions, large amounts of thermal energy are being advected into the central star during an FU Orionis outburst. This should cause the star to expand, which would help to explain why the central stars of FU Orionis objects appear to be twice as large as typical pre-main-sequence stars. The heating and expansion of the star should produce an observational signature: the fading of the central star after the end of rapid disk accretion. Further theoretical explorations of the effects of rapid disk accretion on pre-main-sequence stars are needed.

RP acknowledges the support of grants NASA NAG5-2837 and NSF AST-9423209 at the Center for Astrophysics and grants NASA NAGW-1583, NSF AST 93-15133, and NSF PHY 91-00283 at the University of Illinois. The research of LH and SK is supported in part by NASA grant NAGW-2306.

\section{REFERENCES}

Abramowicz, M. A., Czerny, B., Lasota, J. P., \& Szuszkiewicz, E. 1988, ApJ, 332, 646.

Bell, K. R., \& Lin, D. N. C. 1994, ApJ, 427, 987.

Bell, K. R., Lin, D. N. C., Hartmann, L. W., \& Kenyon, S. J. 1995, ApJ, 444, 376.

Bouvier, J., Cabrit, S., Fernandez, M., Martin, E. L., \& Matthews, J. M. 1993, A\&A, 272, 176.

Bouvier, J., Covino, E., Kovo, O., Martin, E. L., Matthews, J. M., Terranegra, L., \& Beck, S. C. 1995, A\&A, 299, 89.

Cameron, A. C., \& Campbell, C. G. 1993, A\&A, 274, 309.

Choi, P. I., \& Herbst, W. 1996, AJ, 111, 283.

Eaton, N. L., Herbst, W., \& Hillenbrand, L. A. 1995, AJ, 110, 1735.

Edwards, S., et al. 1993, AJ, 106, 372.

Godon, P. 1996, MNRAS, in press.

Hartmann, L. 1994, in Theory of Accretion Disks 2, ed. W. J. Duschl et al. (Dordrecht: Kluwer), p. 19. 
Hartmann, L., Cassen, P., \& Kenyon, S. J. 1996, ApJ, submitted.

Hartmann, L., \& Kenyon, S. J. 1985, ApJ, 299, 462.

Hartmann, L., \& Kenyon, S. J. 1987, ApJ, 312, 243.

Hartmann, L., \& Kenyon, S. J. 1996, ARA\&A, in press.

Hartmann, L., Kenyon, S. J., \& Hartigan, P. 1993, in Protostars and Planets III, ed. E. H. Levy \& J. I. Lunine (Tucson: Univ. of Arizona Press), 497.

Herbig, G. H. 1977, ApJ, 217, 693.

Kenyon, S. J. 1995, Rev. Mex. Astron. Astrofis. (Serie de Conferencias), 1, 237.

Kenyon, S. J., \& Hartmann, L. 1991, ApJ, 383, 664.

Kenyon, S. J., Hartmann, L., \& Hewett, R. 1988, ApJ, 325, 231 (KHH).

Kenyon, S. J., Hartmann, L., Imhoff, C. L., \& Cassatella, A. 1989, ApJ, 344, 925.

Kley, W. 1991, A\&A, 247, 95.

Königl, A. 1991, ApJ, 370, L39.

Larson, R. B. 1983, Rev. Mex. Astron. Astrofis., 7, 219.

Lynden-Bell, D., \& Pringle, J. E. 1974, MNRAS, 168, 603.

Muchotrzeb, B., \& Paczyński, B. 1982, Acta Astron., 32, 1.

Narayan, R., \& Popham, R. 1993, Nature, 362, 820.

Narayan, R., \& Yi, I. 1995, ApJ, 444, 231.

Paczyński, B. 1991, ApJ, 370, 597.

Paczyński, B., \& Bisnovatyi-Kogan, B. 1981, Acta Astron., 31, 283.

Popham, R., \& Narayan, R. 1991, ApJ, 370, 604.

Popham, R. 1996, ApJ, in press (Paper I).

Popham, R., \& Narayan, R. 1995, ApJ, 442, 337.

Popham, R., Narayan, R., Hartmann, L., \& Kenyon, S. 1993, ApJ, 415, L127 (PNHK).

Prialnik, D., \& Livio, M. 1985, MNRAS, 216, 37.

Savage, B. D., \& Mathis, J. S. 1979, ARA\&A, 17, 73.

Shakura, N. I., \& Sunyaev, R. A. 1973, A\&A, 24, 337.

Shu, F. H., Najita, J., Ostriker, E., Wilkin, F., Ruden, S., \& Lizano, S. 1994, ApJ, 429, 781. 
Fig. 1. - Three boundary layer solutions with different values of $\dot{M}$ and $R_{*}$; the solutions have $\dot{M}=10^{-4.3}, 10^{-4.15}, 10^{-4.0} \mathrm{M}_{\odot} \mathrm{yr}^{-1}$, and $R_{*}=2.25,3,4 \times 10^{11} \mathrm{~cm}$, respectively, and are labeled by their values of $R_{*}$ in units of $10^{11} \mathrm{~cm}$. These are the choices of $\dot{M}$ and $R_{*}$ that were used in Paper I; note that they keep the accretion luminosity approximately constant. All three solutions have high angular momentum accretion rates, with $j=1.0,0.9,0.8$, respectively, and all three have $M_{*}=0.5 \mathrm{M}_{\odot}, \alpha=10^{-2}$, and $v_{R, *}=-1000 \mathrm{~cm} \mathrm{~s}^{-1}$. The four panels show (a) the angular velocity $\Omega$; the Keplerian angular velocity for these parameters is shown by the dashed line; (b) the effective temperature $T_{\text {eff }}$; (c) line profiles at 6170 $\AA$, assuming an inclination $i=60^{\circ}$ and instrumental broadening of $12.5 \mathrm{~km} \mathrm{~s}^{-1}$; and (d) blackbody spectra of the disk and boundary layer.

Fig. 2.- The locations in the $\Omega_{*}-H_{*}$ plane of boundary layer solutions with $j=0.9,0$, and -1 (solid lines) and with $v_{R, *}=-1000 \mathrm{~cm} \mathrm{~s}^{-1}$ (dashed line), for $\dot{M}=10^{-4.15} \mathrm{M}_{\odot} \mathrm{yr}^{-1}$, $M_{*}=0.5 \mathrm{M}_{\odot}, R_{*}=3 \times 10^{11} \mathrm{~cm}$, and $\alpha=10^{-2}$. The locations of the five solutions shown in Figure 3 are marked on the $j=0.9$ track; these solutions have $H_{*}=0.8,0.9,1.0,1.1$, $1.2 \times 10^{11} \mathrm{~cm}$. The locations of the five solutions shown in Figure 4 are shown on the $v_{R, *}=-1000 \mathrm{~cm} \mathrm{~s}^{-1}$ track; these solutions (from left to right) have $j=0.94,0.90,0.84$, $0.60,0.0$.

Fig. 3.- Same as Fig. 1, but for five solutions along the $j=0.9$ track shown in Fig. 2, labeled by their values of $H_{*}$. The five solutions have $H_{*}=0.8,0.9,1.0,1.1,1.2 \times 10^{11} \mathrm{~cm}$, and different values of $v_{R, *}$, and all five have $\dot{M}=10^{-4.15} \mathrm{M}_{\odot} \mathrm{yr}^{-1}, M_{*}=0.5 \mathrm{M}_{\odot}$, $R_{*}=3 \times 10^{11} \mathrm{~cm}$, and $\alpha=10^{-2}$.

Fig. 4. - Same as Figs. 1 and 3, but for five solutions along the $v_{R, *}=-1000 \mathrm{~cm} \mathrm{~s}^{-1}$ track in Fig. 2, labeled by their values of $j$. The five solutions have $j=0.94,0.90,0.84,0.60,0.0$, and all five have $\dot{M}=10^{-4.15} \mathrm{M}_{\odot} \mathrm{yr}^{-1}, M_{*}=0.5 \mathrm{M}_{\odot}, R_{*}=3 \times 10^{11} \mathrm{~cm}$, and $\alpha=10^{-2}$.

Fig. 5.- Same as Figs. 1, 3, and 4, but for five solutions with $\log \alpha=-3.0,-2.5,-2.0,-1.5$, -1.0, labeled by their value of $\alpha$. All five solutions have $\dot{M}=10^{-4.15} \mathrm{M}_{\odot} \mathrm{yr}^{-1}, M_{*}=0.5 \mathrm{M}_{\odot}$, $R_{*}=3 \times 10^{11} \mathrm{~cm}$, and $j=0$.

Fig. 6. - A boundary layer and disk solution for FU Orionis. This solution has $\dot{M}=$ $10^{-3.7} \mathrm{M}_{\odot} \mathrm{yr}^{-1}, M_{*}=0.7 \mathrm{M}_{\odot}, R_{*}=4 \times 10^{11} \mathrm{~cm} \simeq 5.75 \mathrm{R}_{\odot}, j=0$, and $\alpha=10^{-2}$. (a) shows the angular velocity $\Omega$ (solid line) and the rotational velocity $v_{\text {rot }}$ (dotted line); the Keplerian angular velocity $\Omega_{K}$ is shown for comparison (dashed line). (b) shows the effective temperature $T_{\text {eff }}$. (c) shows cross-correlations from observed spectra (dashed lines) and line profiles calculated from our boundary layer and disk solution (solid lines) for $i=60^{\circ}$ at $6170 \AA$ (left panel), assuming $12.5 \mathrm{~km} \mathrm{~s}^{-1}$ broadening, and at $2.2 \mu \mathrm{m}$ (right panel), assuming $15 \mathrm{~km} \mathrm{~s}^{-1}$ broadening. (d) shows the blackbody spectrum (solid line) and the 
stellar composite spectrum (dashed line) for this solution, assuming a distance of $500 \mathrm{pc}$; the photometric data for FU Orionis, dereddened assuming $A_{V}=2.2$, are shown as square boxes.

Fig. 7. - Similar to Fig. 6, but for V1057 Cygni. This solution has $\dot{M}=10^{-4.0} \mathrm{M}_{\odot} \mathrm{yr}^{-1}$, $M_{*}=0.5 \mathrm{M}_{\odot}, R_{*}=3.5 \times 10^{11} \mathrm{~cm} \simeq 5.03 \mathrm{R}_{\odot}, j=0$, and $\alpha=10^{-2}$. (a)-(d) are the same as in Fig. 6, except that we have taken $i=30^{\circ}$, a distance of $600 \mathrm{pc}$, and $A_{V}=3.5$, and the $2.2 \mu \mathrm{m}$ profile is broadened by $10 \mathrm{~km} \mathrm{~s}^{-1}$. 

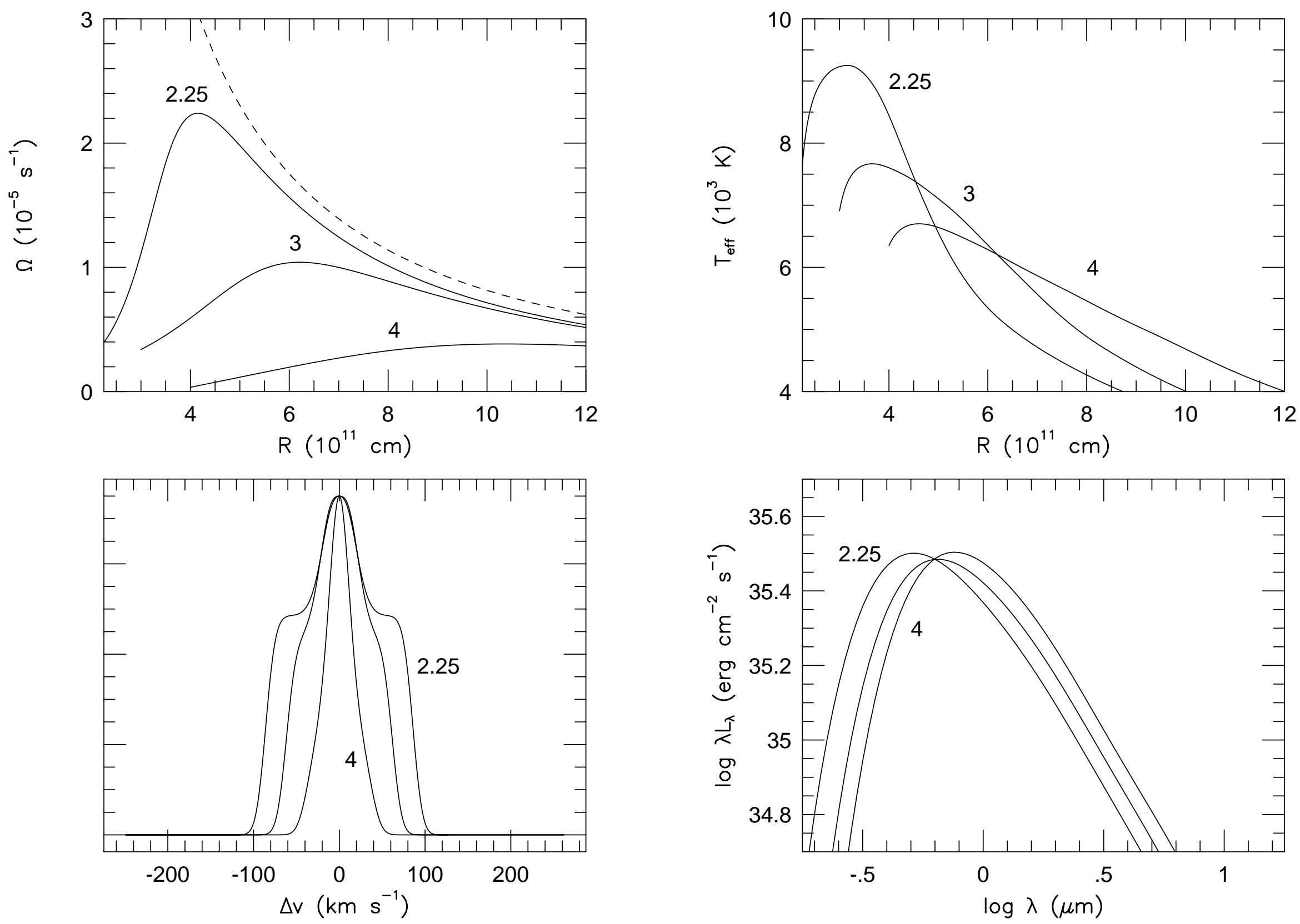


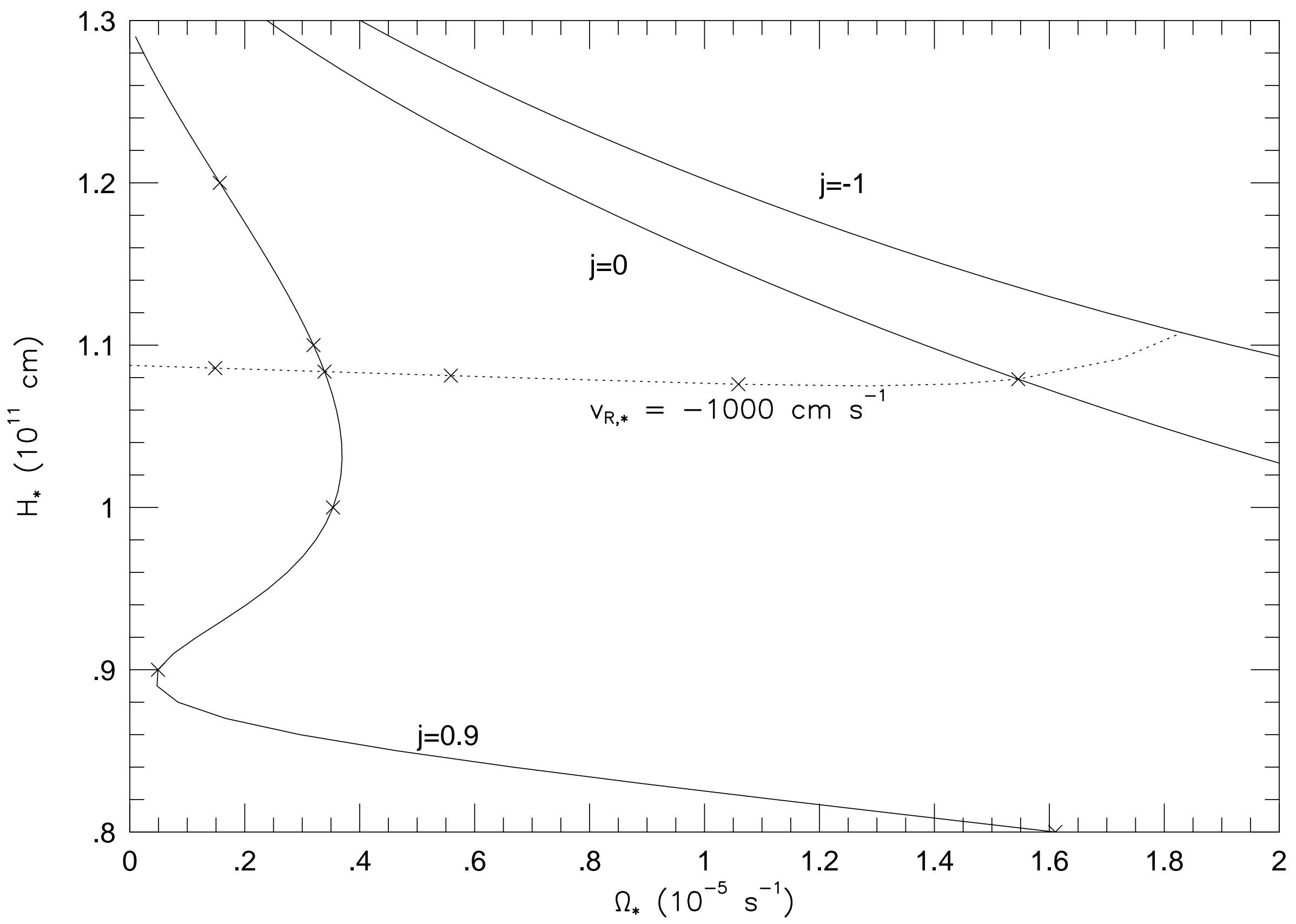



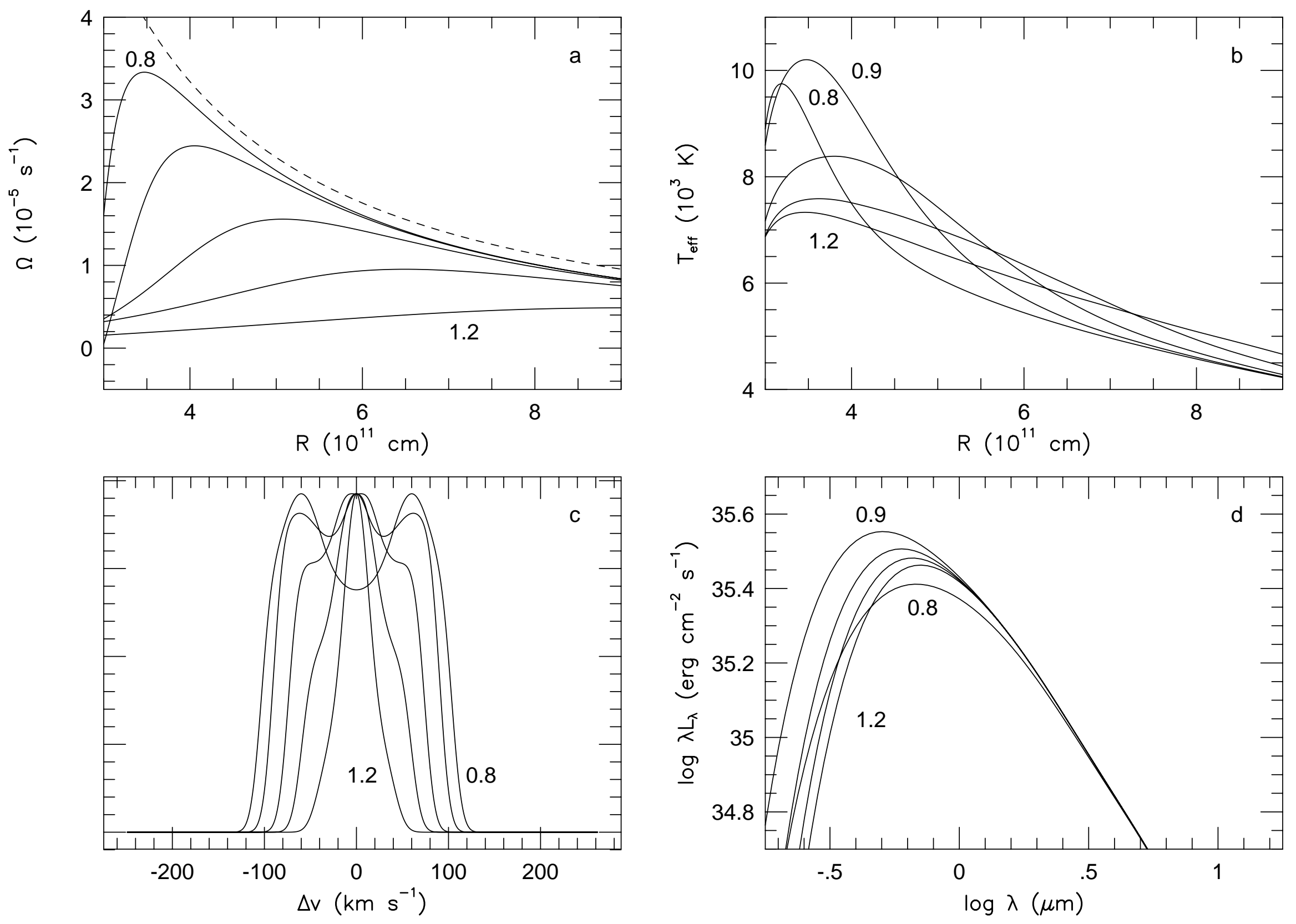

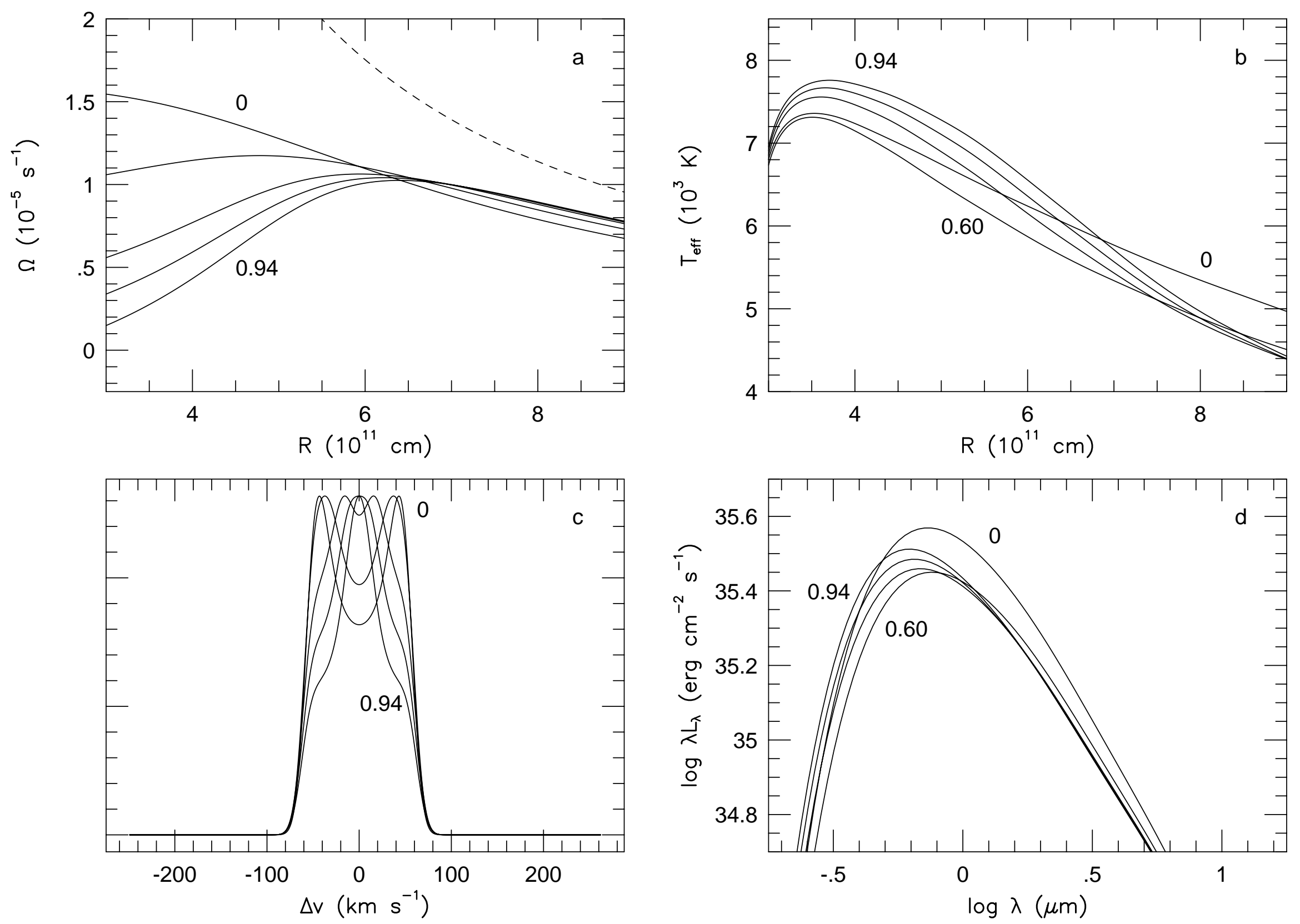

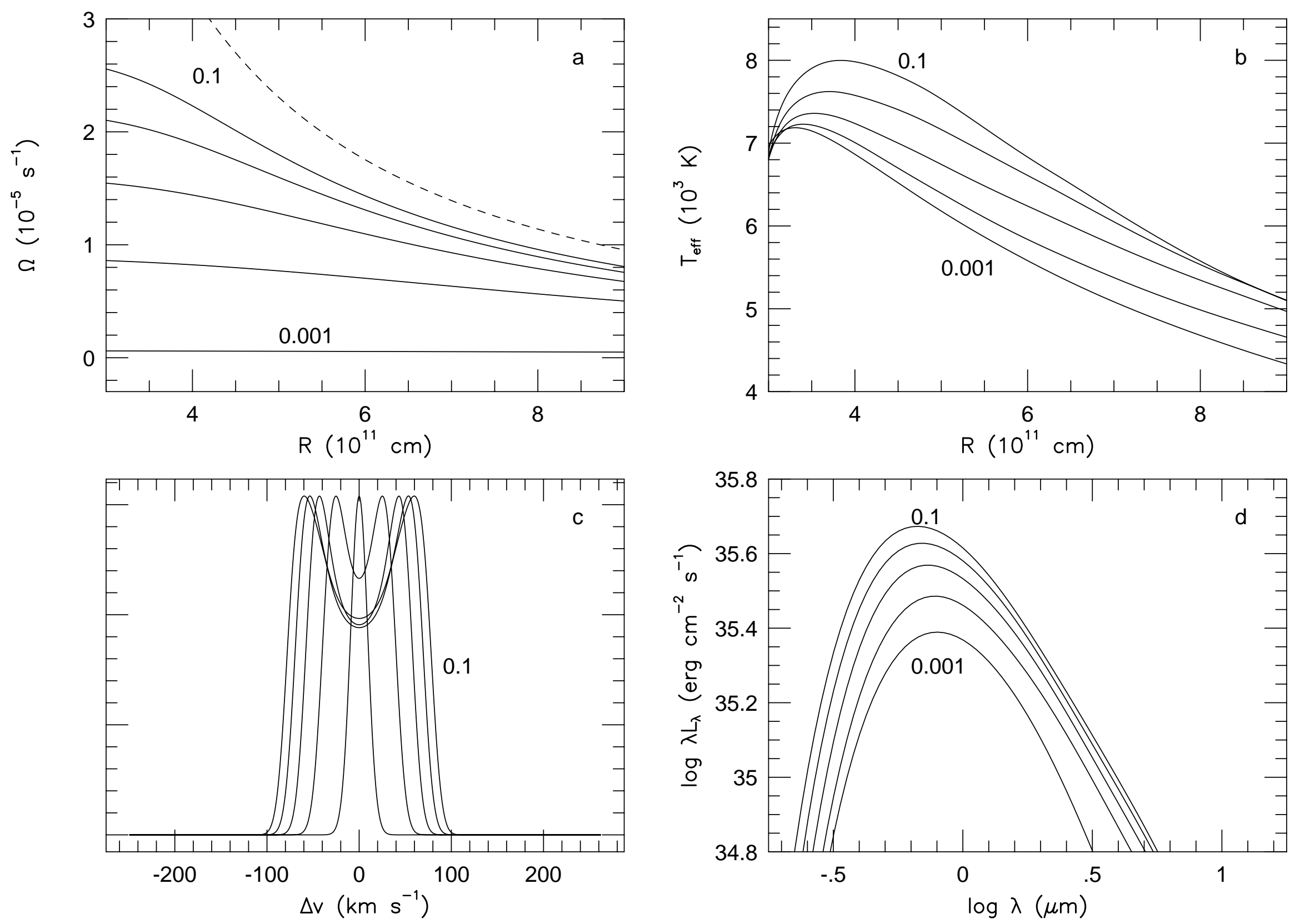


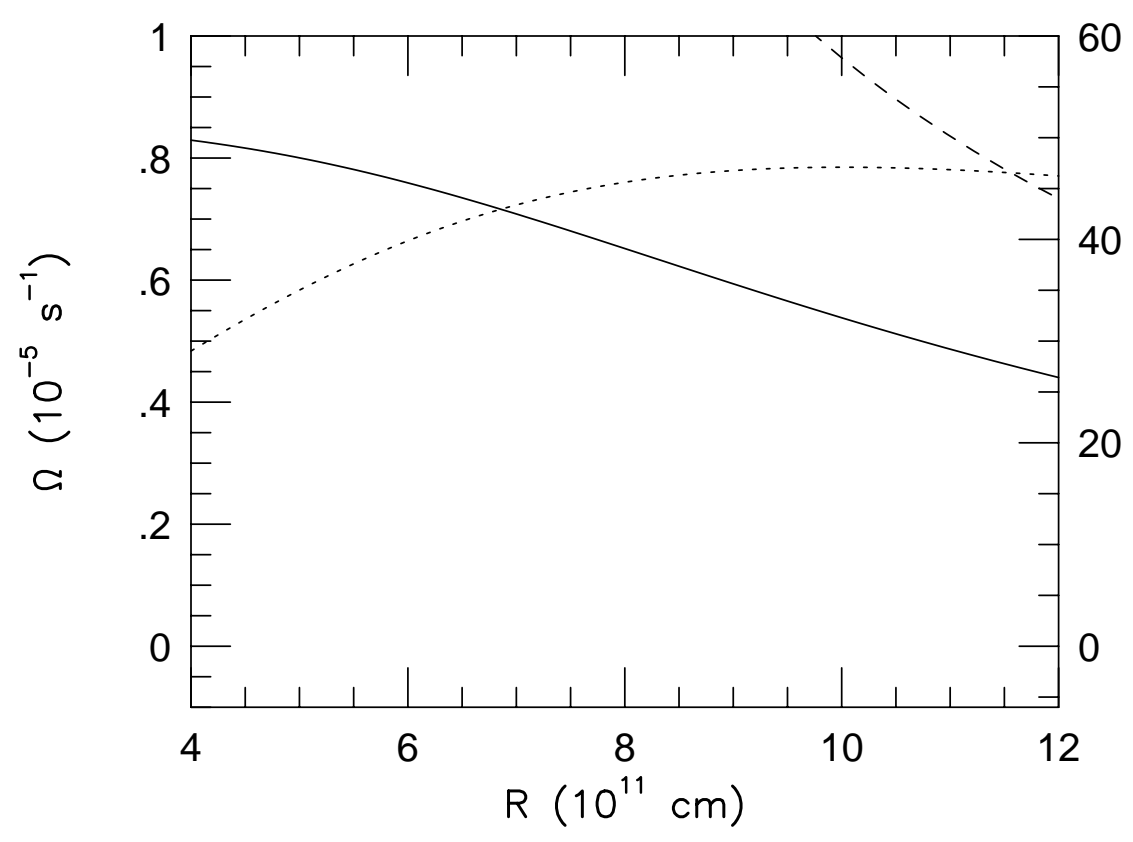

0
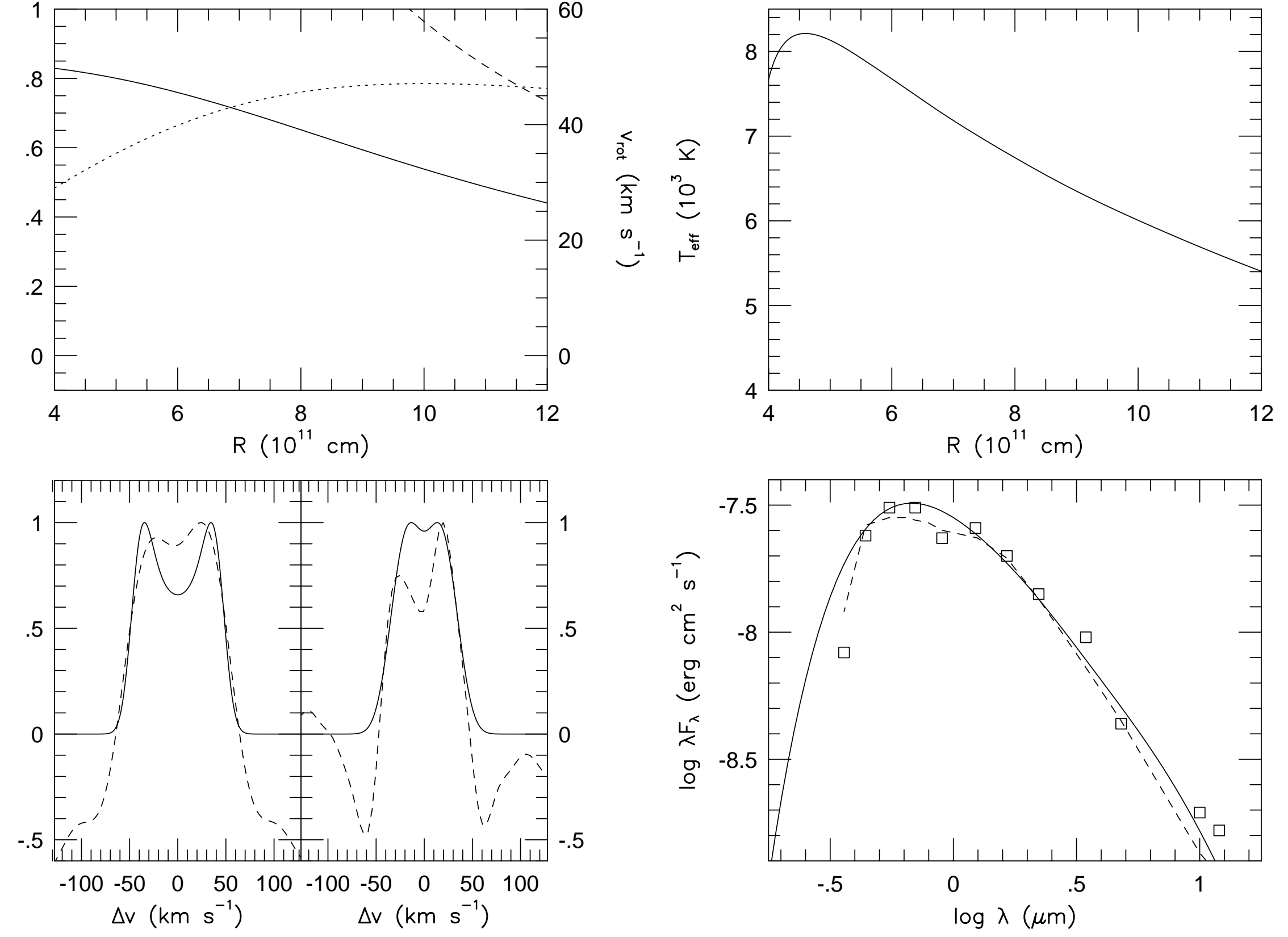

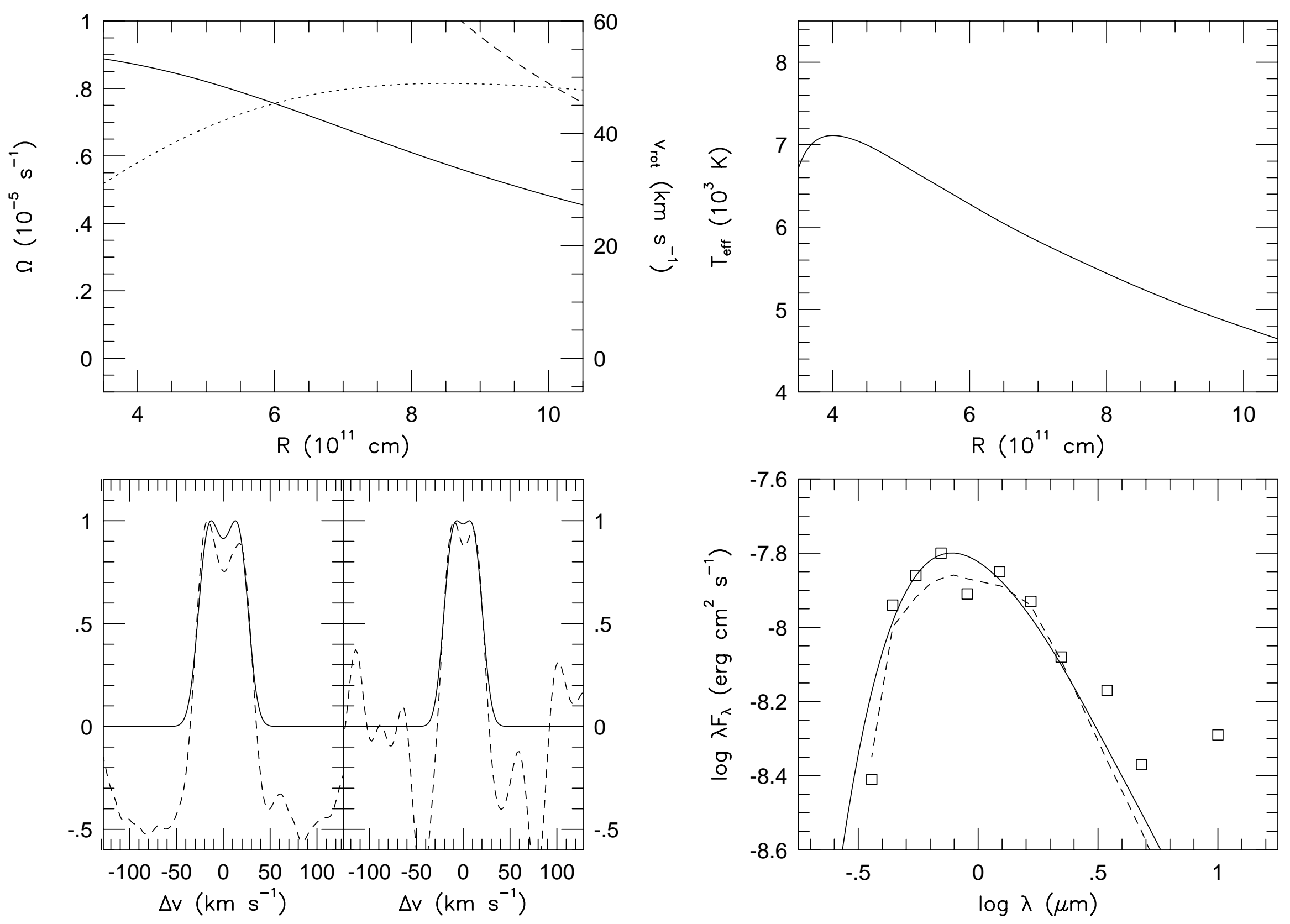